\title{
Chirality sorted single walled carbon nanotubes for random network thin film applications
}

\author{
Volkan Ozyar \\ West Virginia University
}

Follow this and additional works at: https://researchrepository.wvu.edu/etd

\section{Recommended Citation}

Ozyar, Volkan, "Chirality sorted single walled carbon nanotubes for random network thin film applications" (2012). Graduate Theses, Dissertations, and Problem Reports. 4906.

https://researchrepository.wvu.edu/etd/4906

This Thesis is protected by copyright and/or related rights. It has been brought to you by the The Research Repository @ WVU with permission from the rights-holder(s). You are free to use this Thesis in any way that is permitted by the copyright and related rights legislation that applies to your use. For other uses you must obtain permission from the rights-holder(s) directly, unless additional rights are indicated by a Creative Commons license in the record and/ or on the work itself. This Thesis has been accepted for inclusion in WVU Graduate Theses, Dissertations, and Problem Reports collection by an authorized administrator of The Research Repository @ WVU. For more information, please contact researchrepository@mail.wvu.edu. 


\title{
CHIRALITY SORTED SINGLE WALLED CARBON NANOTUBES FOR RANDOM NETWORK THIN FILM APPLICATIONS
}

\author{
by \\ VolkanOzyar \\ Thesis submitted to the College of Engineering and Mineral Resources \\ At West VirginiaUniversity \\ in partial fulfillment of the requirements \\ for the degree of \\ Master of Science \\ in \\ Mechanical Engineering
}

Approved by

DaneeshSimien, Ph.D., Committee Chairperson

Clayton Simien, Ph.D.

Xueyan Song, Ph.D.

Department of Mechanical and Aerospace Engineering

Morgantown, West Virginia

2012

Keywords: Single walled carbon nanotubes; percolation threshold.

@2012 OzyarVolkan 


\section{Abstract \\ CHIRALITY SORTED SINGLE WALLED CARBON NANOTUBES FOR RANDOM NETWORK THIN FILM APPLICATIONS}

\section{VolkanOzyar}

Single-walled carbon nanotubes (SWNTs) are comprised entirely of carbon atoms which lie in a graphitic plane rolled into a seamless cylinder. The direction in which the graphic plane is rolled affects the subsequent chiralities of the SWNTs. This direction is called the chiral vector. This chiral vector, $\mathrm{C}_{\mathrm{h}}=\mathrm{na}_{1}+\mathrm{ma}_{2}$, is described in terms of a pair of integers $(n, m)$ and a pair of lattice vectors $\left(a_{1}, a_{2}\right)$ associated with each carbon atom in the graphene sheet. The magnitude of the chiral vector determines the diameter of the nanotubes as $\mathrm{d}=\mathrm{C}_{\mathrm{h}} / \pi$. SWNTs with integers $\mathrm{n}-\mathrm{m}=3 i$, where $i=0,1,2,3 \ldots$ are metallic at room temperature, while those with $\mathrm{n}-\mathrm{m}=3 i+1$ and $\mathrm{n}-\mathrm{m}=3 i+2$ are semiconducting at room temperature.

SWNTs are being incorporated into a myriad of materials for applications ranging from biosensors to nanoscale electronics to structural materials. The ability to produce SWNTs with well definedchiralities will enable us to conduct more fine tuned experiments with even better reproducibility of our results. As-produced SWNTs grown using the most common synthesis methods of arc discharge, laser ablation and chemical vapour deposition techniques, each render nanotube powders with varying amounts of catalysts and uniformly presents SWNTs with a variety of chiralities and lengths. None of these procedures has yet to produce a single chirality SWNT powder, therefore developing techniques to produce monodispersed SWNT fractions via post-synthetic approaches are essential to have truly reproducible studies where SWNT are incorporated. In this thesis, we present our efforts to sort cobalt molybdenum catalyst grown nanotubes (CoMoCAT) by chirality, which are rich in $(6,5)$ nanotubes species, identifying homogeneous fractions of single chirality $(6,5)$ nanotubes and a secondary mixed layer of $(6,5)$ and $(7,5)$ using density gradient ultracentrifugation techniques. Additionally these individualized species were deposited as random network thin films 
on cellulose ester membranes and evaluated for their critical percolations concentration (onset of electrical conductivity), and surface conductivity using the Generalized Effective Media (GEM) model. 


\section{ACKNOWLEDGEMENTS}

I would like to thank my advisor Dr. DaneeshSimien for giving me the opportunity to work with her, for his support, patience, dedication and help in this work. Also, I would like to thank the members of my graduate committee, Dr. Clayton Simien and Dr. Xueyan Song,for their valuable help and advice. I also want to give thanksto team member of the Lab at WVUSaiPraneethGunturuand all my friends for their company and advice. Finally, I thank God for giving me the strength and health to accomplish this stage of my life. I would like to dedicate this work to my family, my

friend Brad Wright, my brother Oguzhan, for giving me their support and strength to accomplish this stage of my life. 


\section{TABLE OF CONTENTS}

\section{Contents}

- CHAPTER 1 - ..................................................................................................................................................1

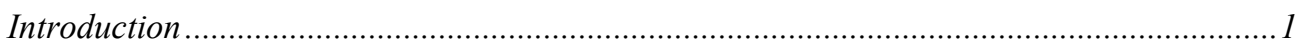

- CHAPTER 2 -

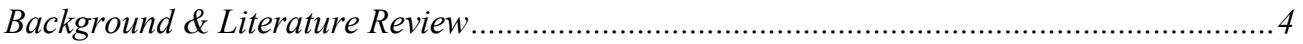

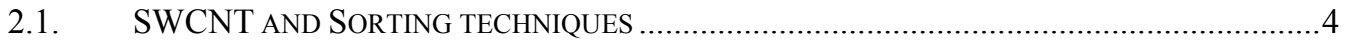

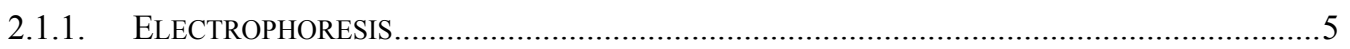

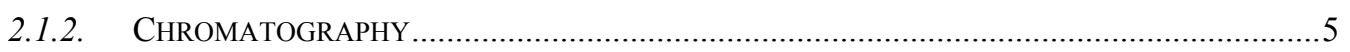

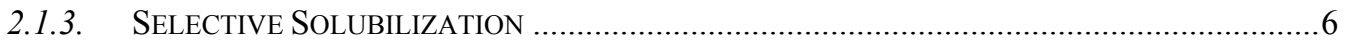

2.1.4. SELECTIVE REACTION.............................................................................................

2.2. DENSITY GRADIENT ULTRACENTRIFUGATION _...............................................................

2.2.1. DENSITY GRADIENT ULTRACENTRIFUGATION WITH THE DIFFERENT SURFACTANTS.......9

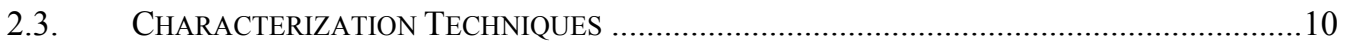

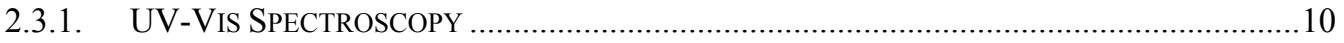

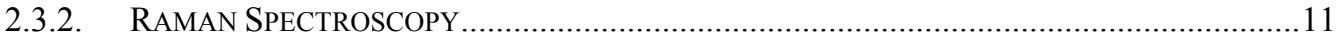

- CHAPTER 3 -

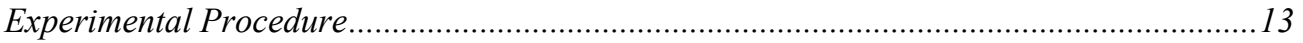

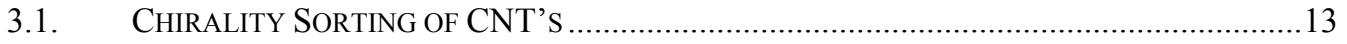

3.2. PINK LAYER VS GREEN LAYER RAMAN AND UV-VIS STUDIES ........................................15

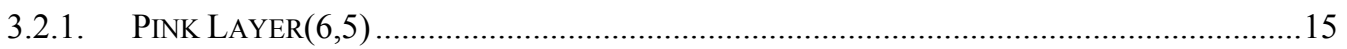

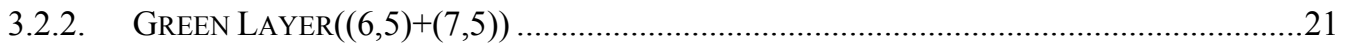

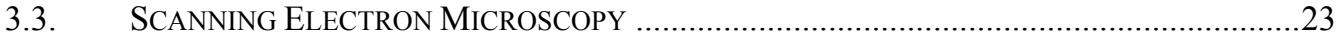

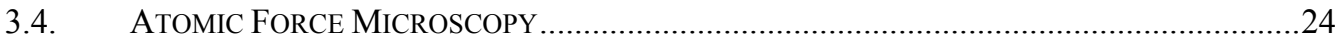

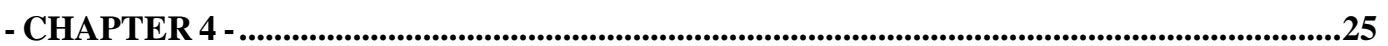

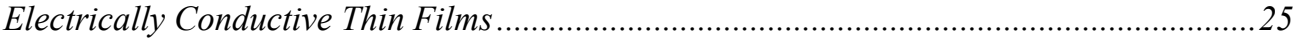

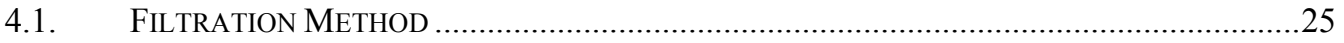

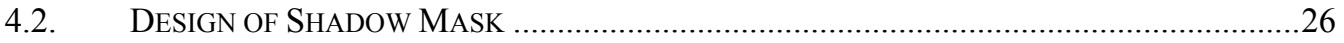

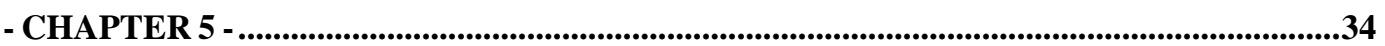

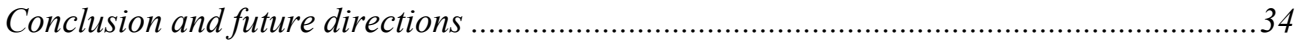




\section{TABLE OF FIGURES}

Figure 1-1 Forming SWCNT by rolling graphene along a chiral vector [7] ................. 1

Figure 1-2 Classification of carbon nanotubes [13]..................................................... 2

Figure 1-3 The mirror image of graphene[11] ...................................................... 2

Figure 2-1 A structure model of DNA-wrapped carbon nanotube[30]............................. 6

Figure 2-2Schematic of surfactant encapsulation and sorting, where $\rho$ is density[36] ...... 7

Figure 2-3 Image of centrifuge tube after separation with using density gradient

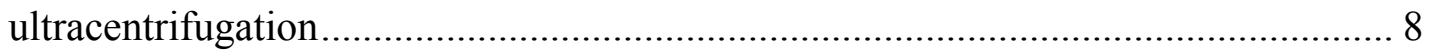

Figure 2-4 The yield of co surfactant separation[39].............................................. 9

Figure 2-5 UV-Vis Spectroscopy of SWCNTs with the metallic and semiconducting

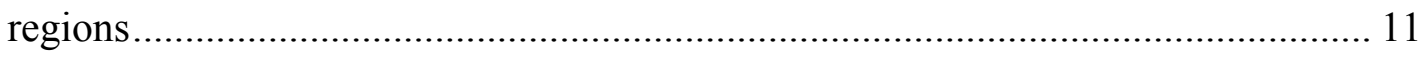

Figure 2-6 Raman Spectroscopy of SWCNTs with different features ............................ 12

Figure 2-7 The relation between RBM frequencies versus nanotube diameter (dashed curve for SWCNT bundles solid curve for isolated SWCNT's)[47]........................ 12

Figure 3-1Ultracentrifuge tube before centrifugation................................................... 13

Figure 3-2 Concentration and the layers of the tube before and after centrifugation....... 14

Figure 3-3 Ultracentrifuge tube after centrifugation................................................... 14

Figure 3-4 Unsorted SWCNTs UV-Vis Graph ...................................................... 15

Figure 3-5 UV-Vis graph of the first layer of the tubes............................................... 16

Figure 3-6 The $1^{\text {st }}$ iteration of the second layer of the centrifuge tubes .......................... 17

Figure 3-7 The $1^{\text {st }}$ iteration of the second layer of the centrifuge tubes ........................... 17 
Figure 3-8 UV-Vis graph of the first layer of the tubes with the second iteration 18

Figure 3-9 UV-Vis graph of the second layer of the tubes with the second iteration....... 18

Figure 3-10 UV-Vis graph of the first layer of the tubes after the third iteration............ 19

Figure $\quad 3-113^{\text {rd }}$ iteration second and the third layer of the tubes UV-Vis graph......... 19

Figure 3-12 Raman spectroscopy of unsorted SWCNTs ........................................ 20

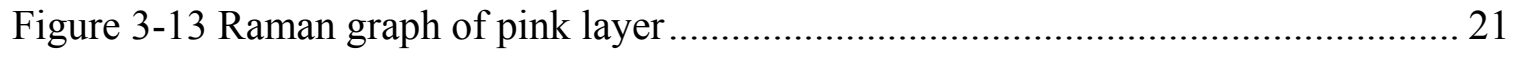

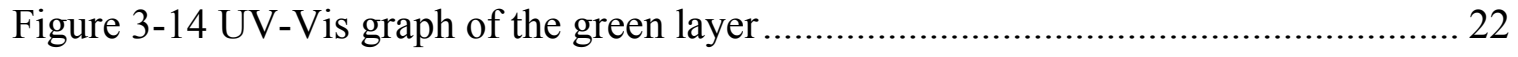

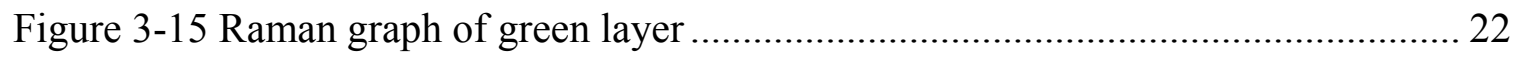

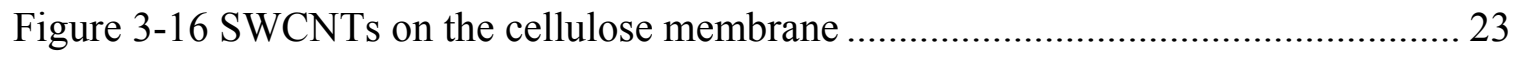

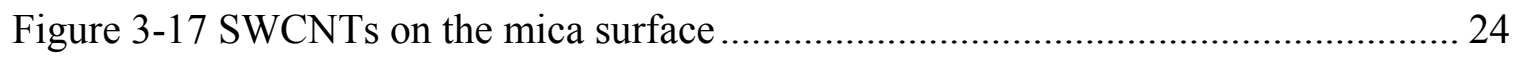

Figure 4-1 Drawings membrane and nanotube layer on the membrane ......................... 25

Figure 4-2 Cellulose membrane and nanotube layer on the membrane.......................... 26

Figure 4-3 Dimensions of the aluminum shadow mask............................................ 27

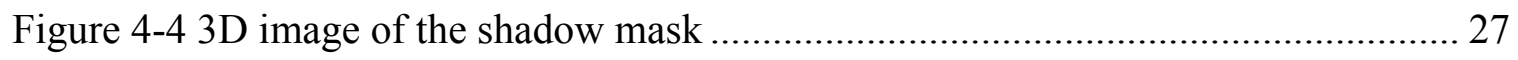

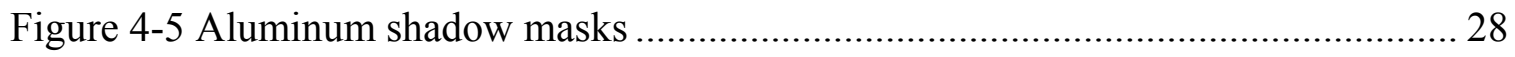

Figure 4-6 Gold sputtered cellulose membranes ................................................. 28

Figure 4-7 Gold sputtered SWCNT network ........................................................ 29

Figure 4-8 Demonstration of the using tweezer type test fixture ................................ 30

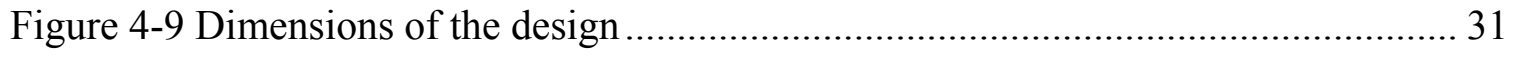


Figure 4-10 A two-dimensional schematic of the percolation of randomly-dispersed high

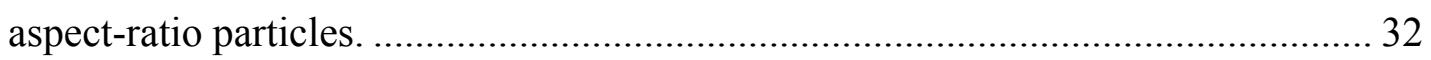

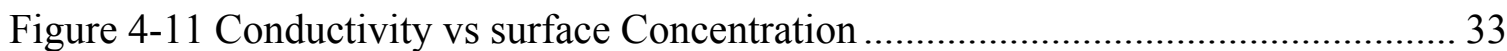




\section{LIST OF TABLES}

Table 1.1.SWCNTs properties............................................

Table 4.1. Green and pink samples surface concentration values...................29

Table 4.2 Impedance values of different samples...................................

Table 4.3.Green vs pink layer conductivity and surface concentration Values.........31 


\section{LIST OF SYMBOLS}

SWCNTsSingle walled Carbon Nanotubes

SsDNASingle StrandedDeoxyribonucleic acid

SEC Size Exchange Chromatography

IEC Ion Exchange Chromatography

DGU Density Gradient Ultracentrifugation

CE Capillary Electrophoresis

SDBS Sodium dodecylbenzenesulfonate

HiPCo High Pressure Carbon Monoxide

DNA Deoxyribonucleic acid

SC Sodium Cholate

SDS Sodium Dodecyl Sulphate

UV-VisUltraviolet Visible Spectroscopy

RBMRadial Breathing Mode

CNFET Carbon Nanotube Field Effect Transistor

PSDPower Spectral Density

SEMScanning Electron Microscope

AFMAtomic Force Microscopy

ComoCATCobalt Molybdenum Catalyst 


\section{- CHAPTER 1 -}

\section{Introduction}

Carbon nanotubes have gotten much attention as an electronic material since their discovery in 1990 by Iijima[1]. Their exceptional properties such as high current carrying capabilities and a high sensitivity to certain molecular adsorbates make them a possible candidate for nanoelectronic applications[2-3]. They have been studied by many researchers and scientists in the area of microscopic thin-film transistors, field-emission sources and chemical and biological sensors[3].

There are three main type of nanotube exist: Multi walled carbon nanotubes, double walled carbon nanotubes and single walled carbon nanotubes.

Carbon nanotubes are molecular-scale tubes of graphitic carbon with outstanding properties. They are among the stiffest and strongest fibres known, and have remarkable electronic properties and many other unique characteristics. Table1.1.shows some properties of SWCNTs.

Table 1.1. SWCNTs properties[4][5][6]

\begin{tabular}{|l|l|l|l|}
\hline & Thermal Conductivity & Youngs Modulus & Electrical Current Density \\
\hline SWCNT & $6.6 \times 10^{4} \mathrm{~W} / \mathrm{mK}$ & $1.25 \mathrm{Tpa}$ & $4 \times 10^{9} \mathrm{~A} / \mathrm{cm}^{\wedge} 2$ \\
\hline
\end{tabular}

The SWNT atomic structure is defined by a twodimensional chiral vector whose components are specified by a pair of positive integers: $(n, m)$. This chirality of the SWNT dictates its properties. Figure 1-1 shows how to graphene become SWCNTs

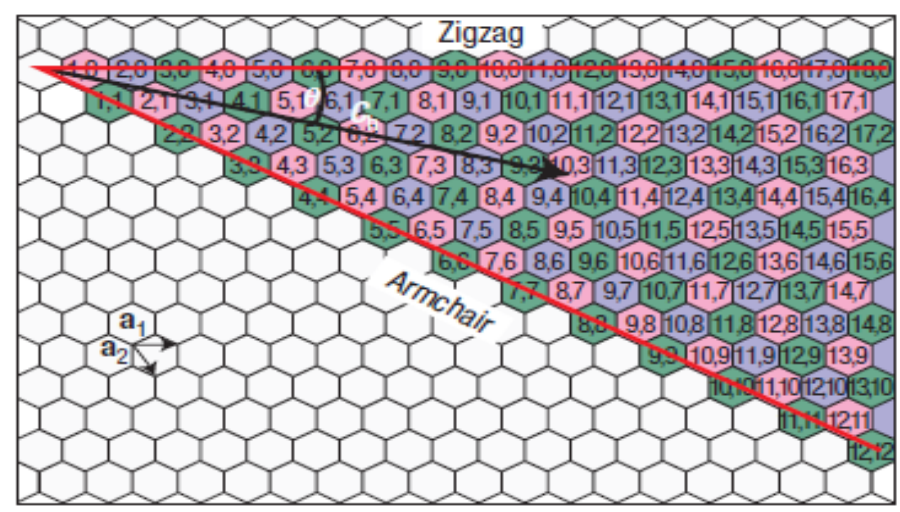

Figure 1-1Forming SWCNT by rolling graphene along a chiral vector [7]. 
There are three main configurations of nanotubes which are armchair, zigzag and chiral. The armchair nanotubes are always metallic and zigzag nanotubes $(n, 0)$ are only metallic when $n$ is a multiple of 3.Chiral nanotubes are metallic when $n-m$ is a multiple of 3[8-9]. Figure 1-2 shows the three main configurations of SWCNTs. Carbon atoms helicity and the diameter of the tube indicate the electronic density of the state and unique electronic character for the nanotubes[10].

a) Armchair: $(5,5)$

b) Zigzag:(9,0)

c) Chiral nanotubes:(10,5)

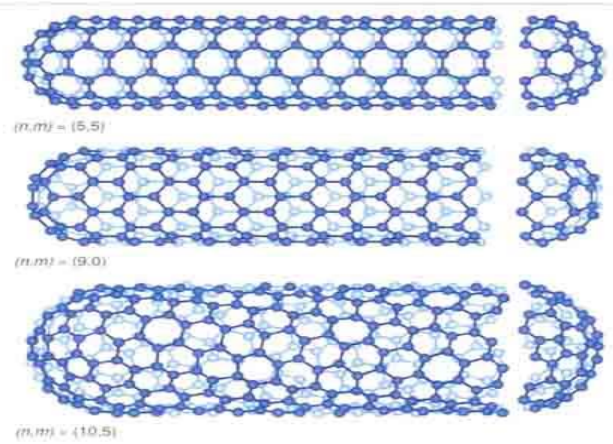

Figure 1-2Classification of carbonnanotubes [13]

Another important feature of SWCNTs are handedness. The handedness of chiral SWNTs is defined as $M$ and $P$. Single layers of graphene sheet cannot be superposed on their mirror-image in the case of chiral SWNTs, while they can be superposed in zigzag and armchair types. Figure 1-3 shows the mirror image of graphene.

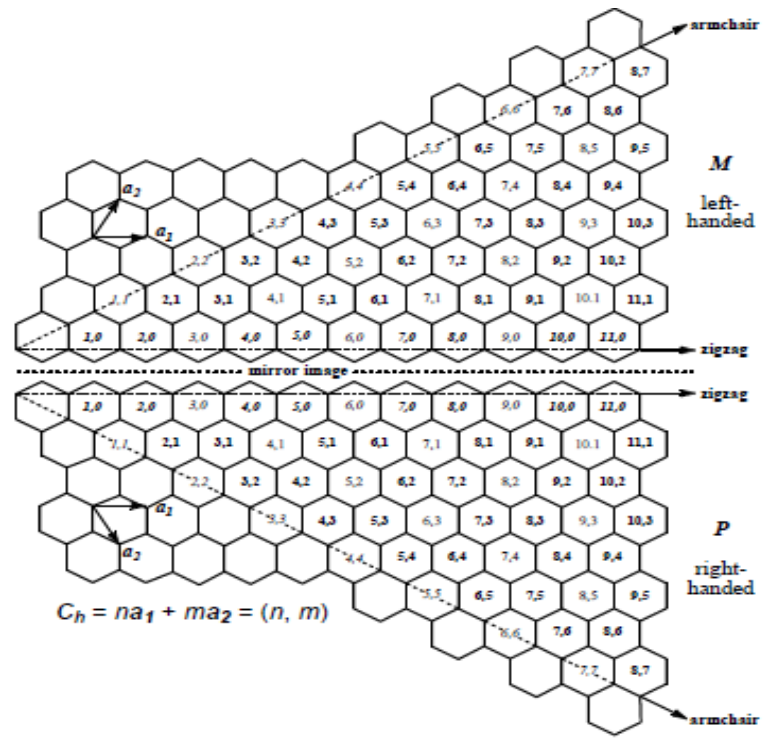

Figure 1-3The mirror image of graphene[11] 
Single wall carbon nanotubes have a very high Young's modulus and high tensile strength and high mobilities[12-13].Those superior properties make them a very good candidate for a lot of applications especially electronic applications, but there are several issues to overcome for using them in electronic applications. One problem is that SWCNT's have a strong tendency to aggregate which is not an ideal case for electronic applications of carbon nanotubes because they exhibit superior electronic properties when they are individualized[10]. 


\section{- CHAPTER 2 -}

\section{Background \& Literature Review}

\subsection{SWCNT and Sorting techniques}

Carbon nanotubes were discovered by SumioIijima in 1991.After the discovery of carbon nanotubes, there have been enormous interest and improvements of the production techniques in this field.[12].

After the discovery of carbon nanotubes, scientists suggested several applications for carbon nanotubes. Those are :electron field emitters,[14], SWCNT attached Atomic Force Microscope tip[15],Lithium and hydrogen storage[16], efficient supports in heterogeneous catalysis[17],microelectrodes in electrochemical reactions[10], Thermoelectric materials[18], Conducting polymer composites[19].

There are certain techniques to produce SWCNTs, such as arc discharge[20], laser ablation[21] and chemical vapor deposition[22]. Even though the production processes has existedfor more than 20 years, there is no control for producing single chirality of SWCNTs. To gather high performance from SWCNT applications, population of SWCNT's should be monodispersed or even beter using single chirality tubes. It is not preferable to use both the metallic and semiconducting nanotubes together. Most of the applications require only one type nanotubes either metallic or semiconducting[23].There are several ways to sort carbon nanotubes by chiral vector, type, diameter and length. However, choosing to correct technique to achieve the final product is essential. The criterias that should be followed are followings:Scalable, compatible nondestructive, iteratively repeatable and affordable[7].

Separation of SWNTs is the way to obtain SWNTs with specific electrical and structural properties, and a lot of methods have been discovered so far for the separation, by diameter, length, chirality $(n, m)$ structure and handedness. Five different methods discovered for the separation process include; electrophoresis, centrifugation, chromatography, selective solubilization and selective reaction[11]. 


\subsubsection{Electrophoresis}

This method is mainly used for separation of metallic and semiconducting nanotubes. In the presence of both metallic and semiconducting nanotubes, metallic CNTs interact with an inhomogeneous electric field and as a result, metallic CNTs being deposited between metal electrodes.This method uses the difference of the relative dielectric constants of the two species with respect to the solvent, resulting in an opposite movement of metallic and semiconducting tubes along the electric field gradient. Metallic tubes goes to the microelectrode array side, leaving semiconducting tubes in the solvent[24].

It has been also demonstrated that capillary electrophoresis (CE) allow separation of carbon nanotubes that are based on differences in length[25]. CE separations depend on charge-density differences between tubes of different geometries, as well as differences in mobility. The total charge on the nanotubes is expected to be proportional to their surface area, and the charge-density differences will be dependent on diameter, so CE method will also allows us to separate nanotubes with different chiralities[26].

\subsubsection{Chromatography}

Chromatography is the technique to separate different molecules and materials from the compound. There are two phases in the compound. One is called mobile phase, which carries it through a structure holding another material called the stationary phase. The various constituents of the mixture travel at different speeds, causing them to separate. This method is applicable for the separation of carbon nanotubes. Carbon nanotube separation mostly two chromatography techniques are used.

1) Size Exclusion Chromatography:SEC is a widely used technique for fractionating macromolecules according to theirphysical sizes. If the molecule is too large or too small,the size-exclusion effect becomes less sensitive, so broad size distribution is necessary to achieve effective SEC. The dominating physical dimension for a CNT is its length, SEC is expected to result in length but not diameter fractionation[27].

2) Ion Exchange Chromatography: DNA-assisted dispersions and separations of SWNTs widely used by the scientists[28]. SWNTs can be dispersed in water with the sonication in the presence of single-stranded deoxyribonucleic acid (ssDNA). Molecular modeling 
suggests that aqueous ssDNA can bind to CNTs through $\pi$-stacking and hydrophobic forces. ssDNA wrapped around a single SWNT and ion exchange chromatography (IEC) of these hybrids separates fractions according to the electronic structure of the SWNT. Figure 2-1 shows how single walled carbon nanotube surrounded by the DNA.It was discovered that certain ssDNA sequences improve IEC separations between both metallic and semiconducting CNTs as well as between semiconducting CNTs of different diameter and electronic band gap[29].

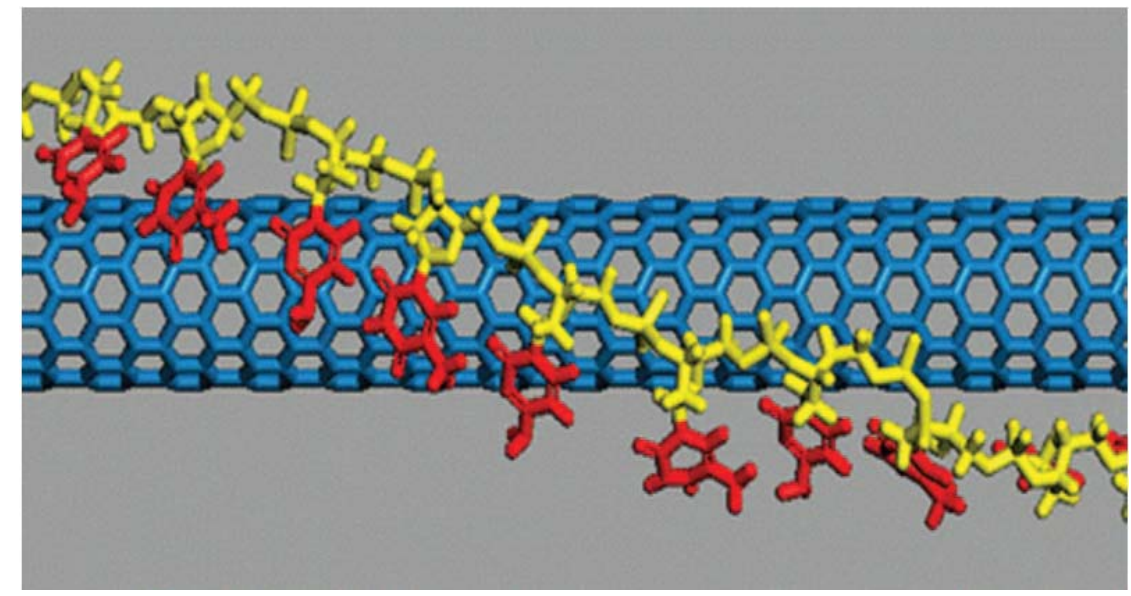

Figure 2-1A structure model of DNA-wrapped carbon nanotube[30]

\subsubsection{Selective Solubilization}

SWNTs are not solubilized in any solvents, but they can be dissolved into solvent in the presence of a solubilizing agent. The solubilizing agents can be alkylamine for metallic/semiconducting separation[31], polymers such as fluorene-based ones for extracting specific $(n, m)$ structure[32], Chen et al.[33] reported that high purity of $(7,5)$ SWNTs can be obtained by extraction from SWNTs produced by Co-MCM-41, but high selectivity cannot be obtained on wide diameter distributed SWNT samples, although some of the polymers can selectively wrap the SWNTs with certain chiral angles or diameters depending on their chemical structures. 


\subsubsection{Selective Reaction}

Most of the selective reactions for SWCNT separation are classified into the following two types of chemical and physical processes; selective oxidation in the presence of some chemicals and selective break-down of either metallic or semiconducting SWNTs by use of electricity, plasma laser microwave and Xe-lamp[11].

Yudasaka et al.[34]have applied light irradiation during the oxidation of SWNTs and this method has enabled the preferential elimination of SWNTs of certain diameters, and semiconductor-type electronic structure, when the light wavelength is chosen to correspond to the gap energy of the SWNTs.

Miyata et al. tested the chirality-dependent combustion of single-walled carbon nanotubes (SWCNTs) during oxidation in both air and hydrogen peroxide. He found out that under airoxidation conditions, SWCNTs with a high chiral angle and smaller diameter decomposemore rapidly[35].

\subsection{Density Gradient Ultracentrifugation}

This technique allows SWCNTs to be sorted by their buoyant density. SWCNT's in the ultracentrifuge tube will match with the density of the gradient after the process. This process used to sort DNA-SWCNT hybrids by diameter, and then used to sort SWCNTs by length, type diameter etc with the different kind of surfactants presence[7]. Figure 2-2 shows how carbon nanotubes were surrounded by the surfactant molecules.

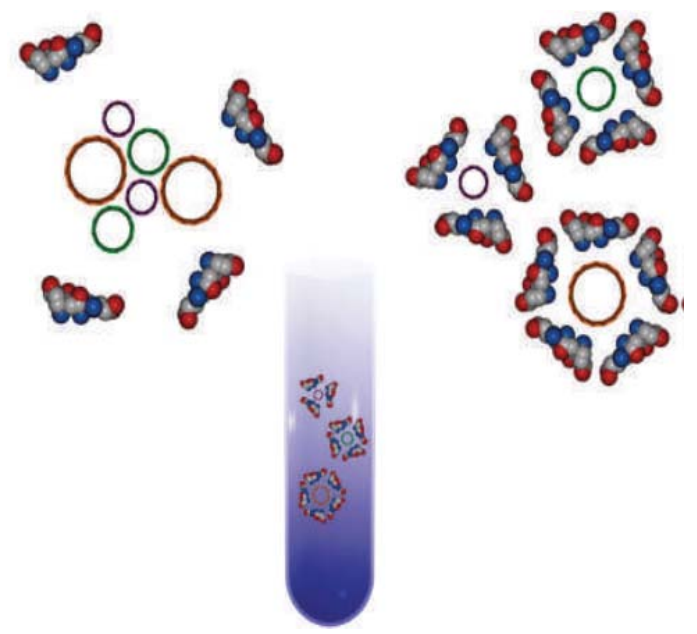

Figure 2-2Schematic of surfactant encapsulation and sorting, where $\rho$ is density[36]. 
Figure 2-3 shows the different layer of sorted single walled carbon nanotubes with the different colors. Different colors are an indication of effective sorting process.

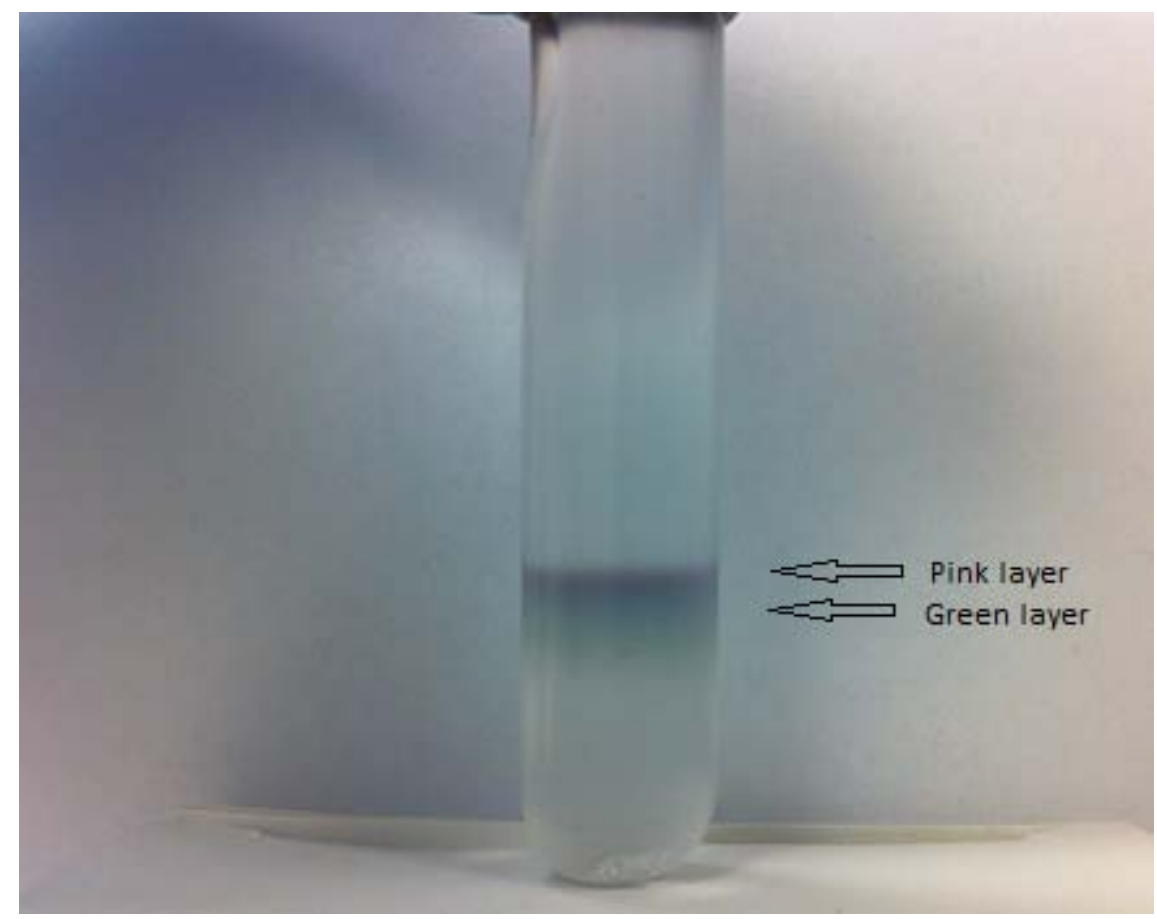

Figure 2-3Image of centrifuge tube after separation with using density gradient ultracentrifugation.

Iodixanol is used as a density gradient medium by many scientists. It has been the most popular density gradient medium for nanotube separation process. There are some disadvantages for using Iodixanol. First of all, iodixanol has iodine atoms, and if those atoms remain in the structure those atoms will act like an electron acceptor for the SWCNT. Second of all, it is a big molecule, and it takes time to remove from the samples. Third of all, it is expensive. Because of those disadvantages, using different density gradient medium, studied by several scientists. Yanagi et al[37]. used sucrose as a density gradient medium. Separation improves when density difference between metallic and semiconducting tubes goes high, and also high viscosity might help the separation between metallic and semiconducting nanotubes. 


\subsubsection{Density Gradient Ultracentrifugation with the different surfactants}

Encapsulating agents have a primary role in sorting process of SWCNT's . In many cases, SWCNT's will interact and bond to each other with the Van der waals forces to form bundles. To understand the relations between SWCNT's and the role of encapsulating agents we need to understand and look at closely to the different encapsulating agents[36]. Different sorting purposes led to using different surfactants.

Inlength separation process deoxycholate is an ideal agent for the seperation and it gives very reliable results[38]. Type separation such as metallic and semiconductive type SWCNT's, co-surfactant agent method is used by Wei et al[39]. Using individual SDS or SC doesn't make any sorting difference for Co-MCM-41 type carbon nanotube, but using 1:4 ratio SDS to SC solution shows significant sorting result on that type of carbon nanotubes. Figure 2-4 shows that to sort $(6,5)$ nanotubes 1:4 ratio is an ideal point. Increasing SC or SDS weight ratio results in poor $(6,5)$ separation. Figure 2-4 shows that using single surfactant for this type of nanotubes is not ideal in terms of chirality separation. After three extraction iterations with SDS and SC cosurfactants Wei et al.[39]achieved $85 \%$ of selective enrichment $(6,5)$ and $(8,3)$ tubes. After getting enough amount of solution Wei used vacuum filtration method to wash away surfactants[39].

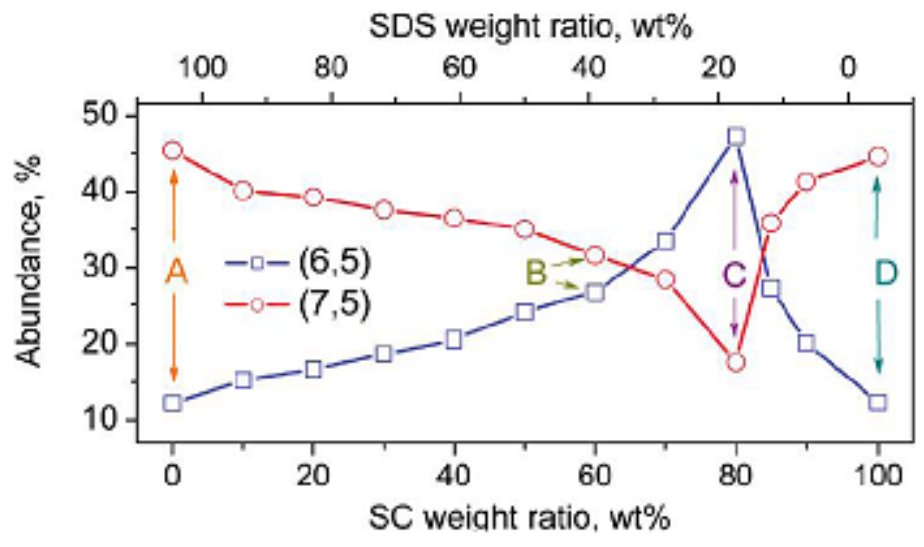

Figure 2-4The yield of co surfactant separation[39] 
This method is only useful for certain type of nanotubes and certain co-surfactant combinations. Mc Donald et al. shows that SDS has a strong binding to the smaller dimater, and SDBS shows weak diameter dependence [40].

Weisman et al. used DGU process for the HiPCo grown SWCNTs. HiPCo type samples contain approximately 30 different semiconducting and metallic species. DGU process with the HiPCo samples are not very easy process because there are a lot of species with very close diameter values. In this study Weisman detected $(10.0)$ and $(11,0)$ species which haven't been detected before in HiPCo samples. In this work, Wei has done enantiomeric separation with 7 different species including $(6,5)$, and characterize this separation with circular dichroism signals of opposite sign at appropriate wavelengths. The different color banding was observed in this process, and achieved to sort samples with only single step DGU separation[41]. The purity of the samples were not as good as different methods used by Zheng[42].

Biswas et al. classified the CNT dispersion in solvent into two groups.Macrodispersion which consist of dozens of CNT bundles and nanodispersion which are individual CNTs. In the presence of SDS and poly in deionized water nanodispersion of SWCNTs can be achieved. Sonication time, surfactant concentration and centrifugation speed are the controllable elements to achieve nanodispersion. Biswas et al. shows that after 10 days sorted nanotubes might lose their stabilization and aggragate. Resonication might be a solution for that problem[43].

\subsection{Characterization Techniques}

There are several tools to prove that dispersion of surfactant and sorting was successfully done. The most common tools are ultraviolet-visible-near-infrared(UV-VisNIR)[38], and Raman Spectroscopy.

\subsubsection{UV-Vis Spectroscopy}

UV-Vis can help us to identify the types and chiralities of SWCNT's by observing the position of the peaks[44].In each system, individual SWCNT will absorb light when the excitation wavelength is similar enough the energy to excite electrons 
from valence band to the conduction band. After that, the peaks can be assigned with the certain chiralities. Peaks between:

400-600 nm correspond S33 of semiconducting SWCNT's

550-900 nm correspond M11 of metallic SWCNT's

800-1600 nm correspond $S 22$ of semiconducting SWCNT's

Figure 2-5 shows the UV-Vis spectroscopy of SWCNTs with S33-M11 and S22 regions.

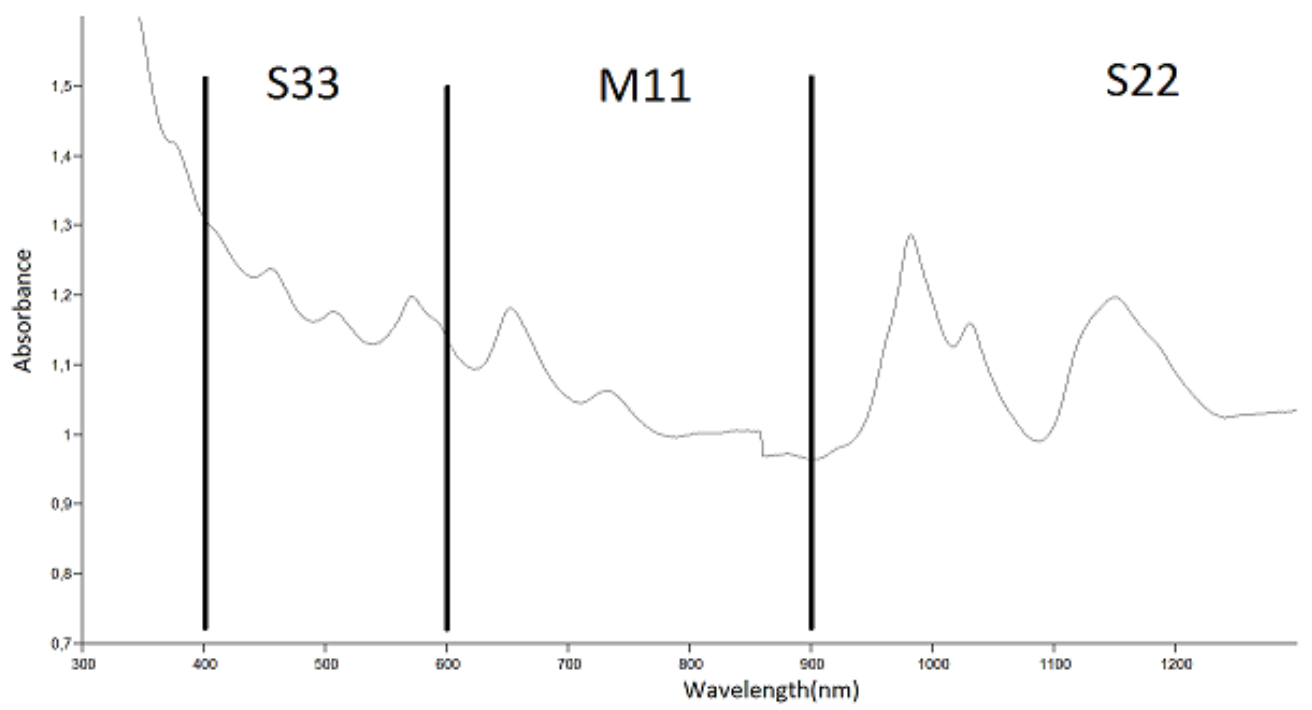

Figure 2-5UV-Vis Spectroscopy of SWCNTs with the metallic and semiconducting regions

These peaks can only be observed when SWCNT's are individually dispersed or in small bundles[45].

\subsubsection{Raman Spectroscopy}

Raman spectroscopy has been used to characterize solid and solution based carbon nanotube systems[46] and they can provide an essential information about the structure and properties of nanotubes[47].There are different modes on Raman spectrum for the SWCNTs such as radial breathing mode (RBM), D mode, G mode and G' mode. Figure 2-6 shows the different mode for SWCNTs. The most important and useful one for characterization is RBM(radial breathing mode). The RBM Raman features between $50 \mathrm{~nm}$ and $400 \mathrm{~nm}$ resemble atomic vibration of the $C$ atoms in the radial direction. 


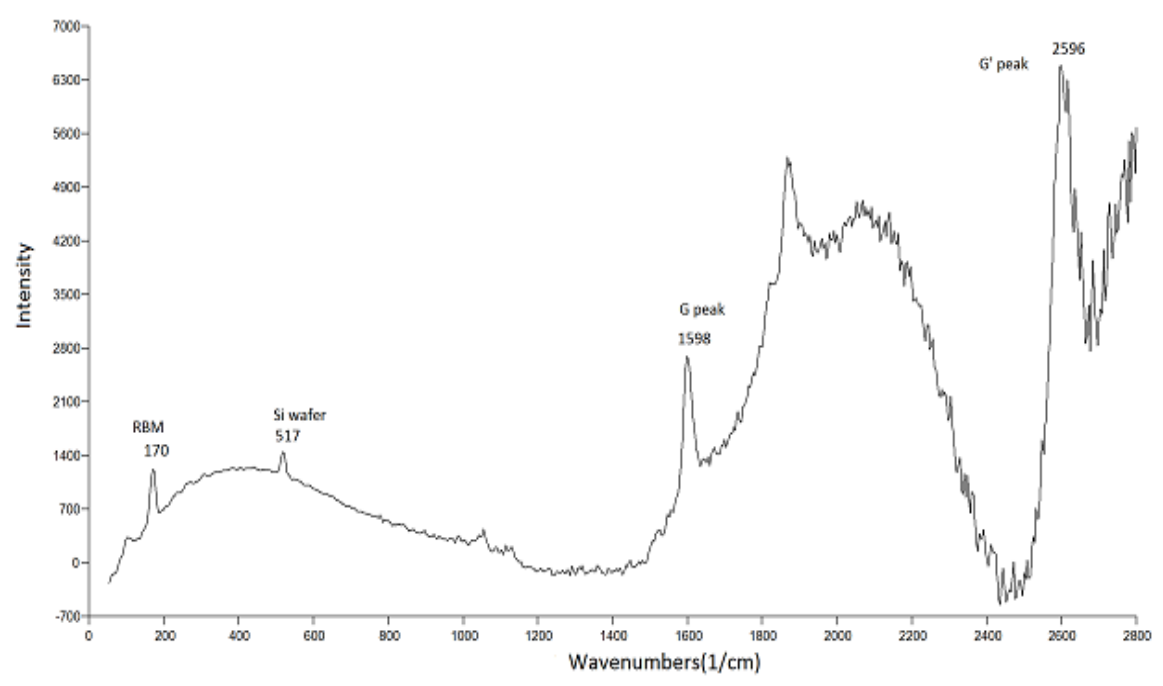

Figure 2-6Raman Spectroscopy of SWCNTs with different features

This mode very useful to assign SWCNT's diameter with the help of RBM peaks.[47] The relations for bundle SWCNTs and isolated SWCNTs are correspondingly,

$$
\begin{gathered}
\omega=A / d_{t}+\mathrm{B} \\
\omega=\mathrm{A} / \mathrm{d}_{\mathrm{t}}+\mathrm{B} \quad \mathrm{A}=234 \mathrm{~cm}^{-1} \mathrm{~B}=10 \mathrm{~cm}^{-1} \\
\mathrm{~A}=248 \mathrm{~cm}^{-1} \mathrm{~B}=0(2)
\end{gathered}
$$

Figure 2-5 shows the relation between RBM frequencies with the diameter of the tube for the bundle SWCNTs and isolated SWCNTs

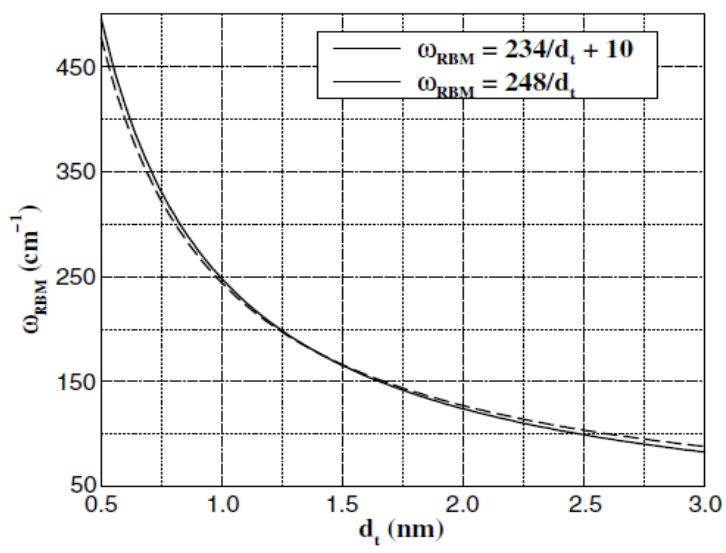

Figure 2-7The relationbetween RBM frequencies versus nanotube diameter (dashed curve for SWCNT bundles solid curve for isolated SWCNT's)[47]. 


\section{- CHAPTER 3 -}

\section{Experimental Procedure}

\subsection{Chirality Sorting of CNT's}

Single-walled carbon nanotubes Cobalt molybdenum catalyst (ComoCAT) were purchased from Southwest Nanotechnologies. For chirality sorting, single-walled carbon nanotubes dispersed at $1 \mathrm{mg} / \mathrm{mL}$ in a $2 \%$ mass fraction of sodium cholate(SC) aqueous surfactant solution via sonication.

There are different solvents with different roles in terms of nanotube separation .Deoxcycholic acid is good for length separation, [48] co surfactant sds-sc is good for chirality sorting[39] and in our case $(6,5)$ tubes separation sodium cholate is the best candidate.

SC used as a surfactant solution because SC binds stronger than the other surfactants to certain chiralities such as $(7,5)$. SC is not a diameter selective surfactant. As an example, $(10,2)$ and $(7,5)$ nanotubes have same diameter, but in the presence of SC $(7,5)$ tube binds strongly to the SC unlike $(10,2)$ nanotubes[49]. The most abundant species in ComoCAT materials are $(6,5)$ tubes[50]. Figure 3-1 shows that the ultracentrifuge tube before ultracentrifugation.

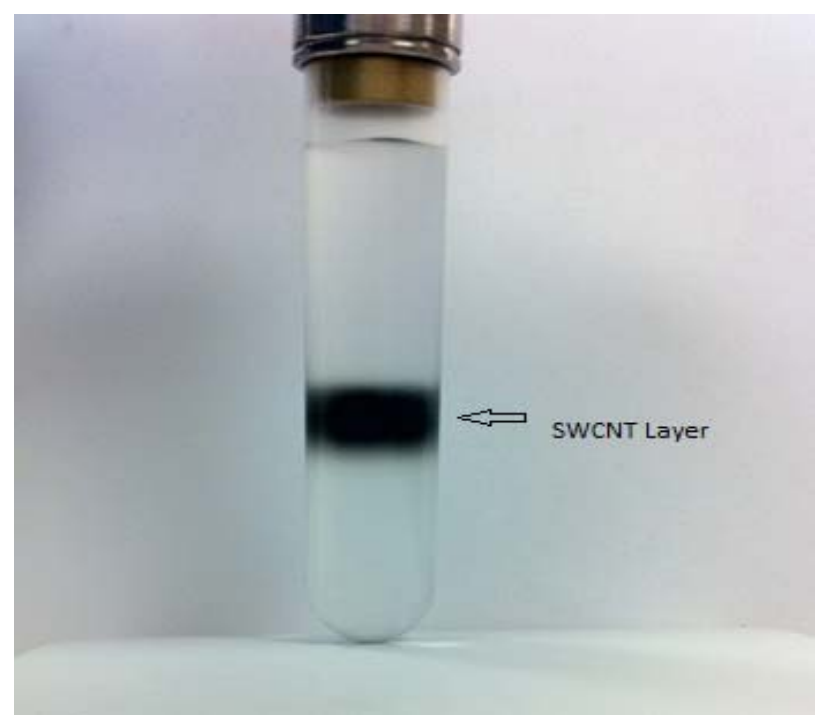

Figure 3-1Ultracentrifuge tube before centrifugation 
After sonication, precentrifugation performed at 50,000 rpm for 21 minutes to remove non-SWNT carbonaceous and catalyst impurities. In order to separate singlewalled carbon nanotubes by their chirality, several solutions have to be prepared with usingdensity gradient solution, iodixanol(OptiPrep, $60 \%$ mass by volume iodixanol) and $2 \%$ sodium chocolate. The bottom of the centrifuge tube is filled with $60 \%$ iodixanol, and one after another $22.5 \%$, SWCNT, $7.5 \%$ and then tube filled with $2 \%$ SC.

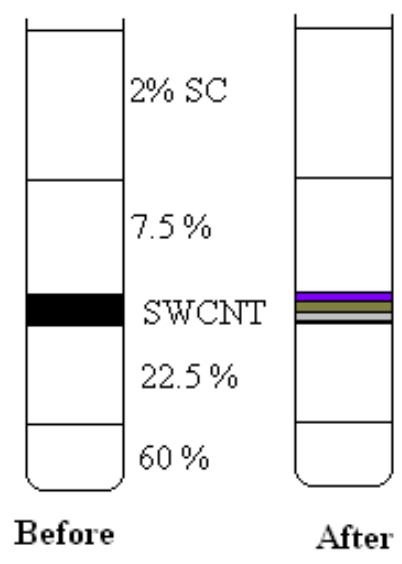

Figure 3-2Concentration and the layers of the tube before and after centrifugation Centrifugation performed at $61,000 \mathrm{rpm}$ at $22^{\circ} \mathrm{Cfor} 4: 26$ hours.After the centrifugation three different colored layers were observed. The changes in optical density and color with respect to the density are a good indication of effective SWNT sorting[51].

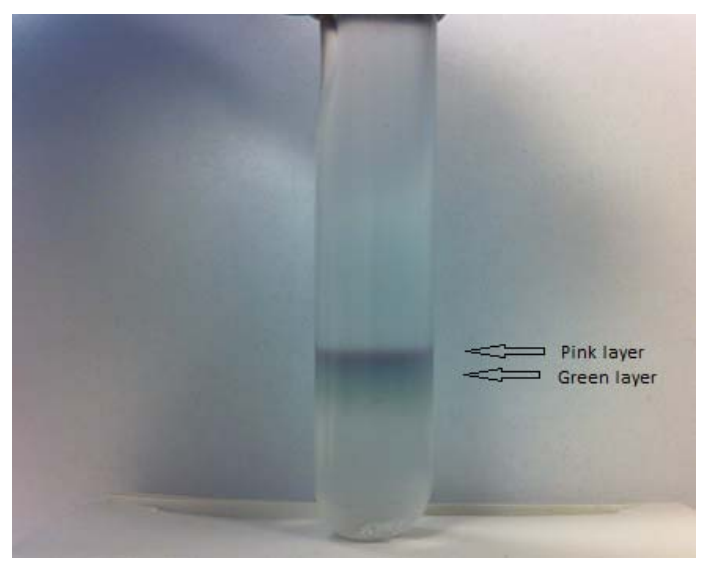

Figure 3-3Ultracentrifuge tube after centrifugation 
Each of the layers were collected. The top layer of the tube was pink in color and the bottom layer was green in color. Each layer was around $100 \mu \mathrm{L}$.

\subsection{Pink layer vs Green layer Raman and UV-Vis Studies}

For characterization of the solution, collection of the sorted tubes are analyzed sample by sample. Figure 3-4 shows UV-Vis graph of unsorted SWCNTs

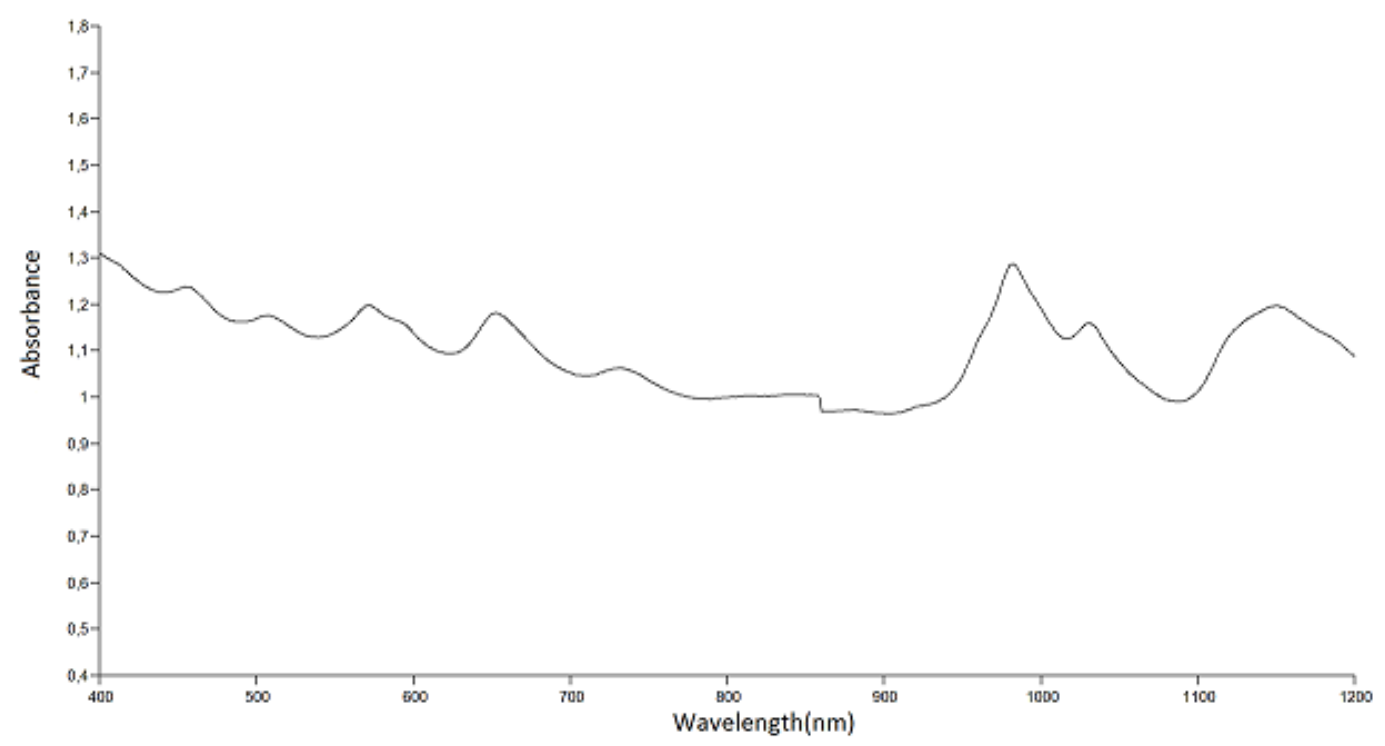

Figure 3-4UnsortedSWCNTs UV-Vis Graph

\subsubsection{Pink Layer $(6,5)$}

The first iteration of centrifugation was run at $61,000 \mathrm{rpm}$ for $4: 26$ hours. This first iteration shows that there is a peak at $571 \mathrm{~nm}$ and $982 \mathrm{~nm}$ and those strong peaks are from $(6,5)$ species. There is also another peak around $1031 \mathrm{~nm}$ and this peak represents $(7,5)$ species which is second dominant species in ComoCAT materials. Figure 3-5 shows the different centrifuge tubes, and their first layer of the UV-Vis Spectroscopy peaks. 


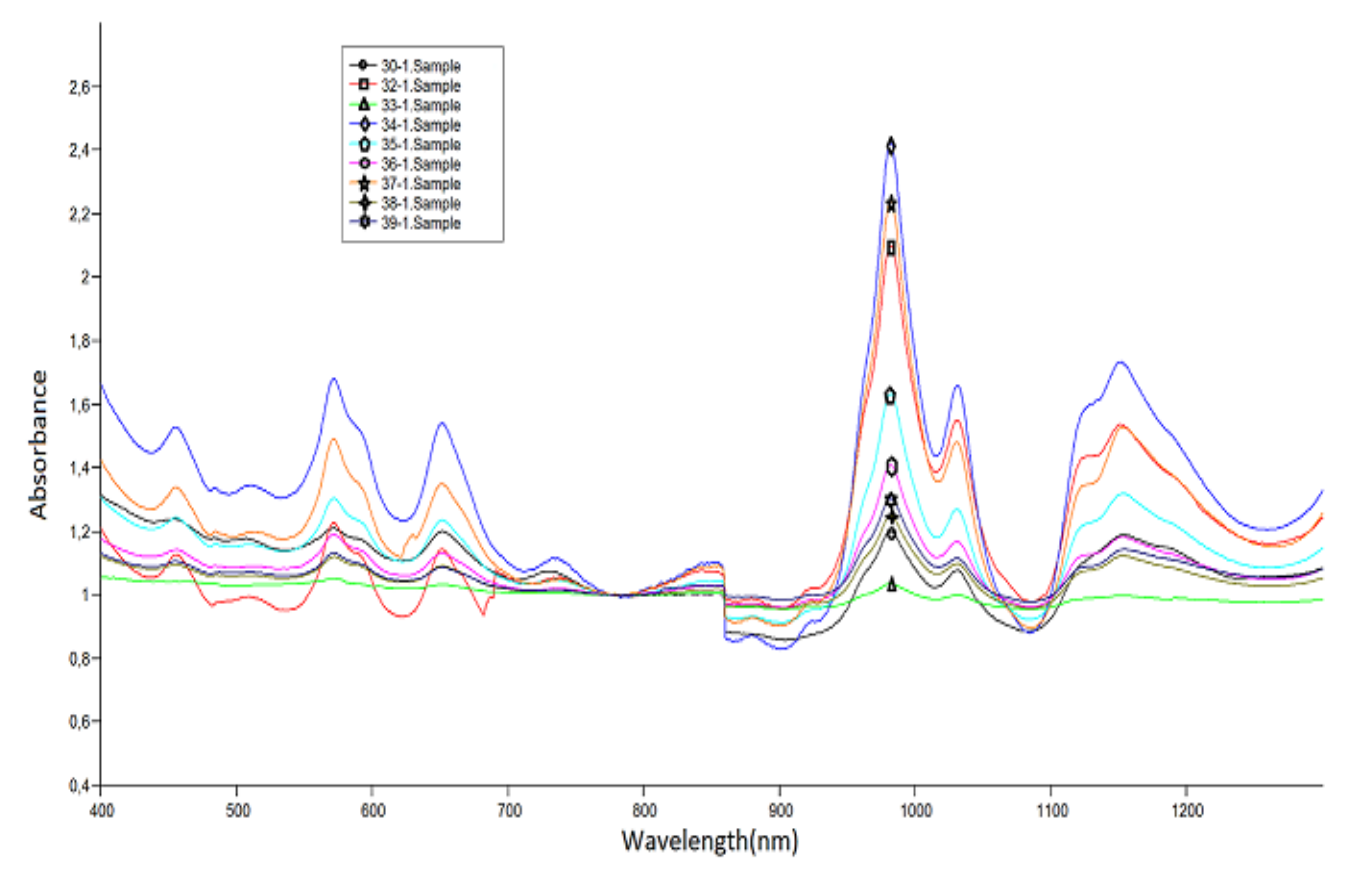

Figure 3-5UV-Vis graph of the first layer of nine centrifuge tubes ran to pre-sort nanotube solution by chirality.

In terms of getting pure $(6,5)$ chiralities, centrifugation was performed with the top layer of the solution again. The second layer and the third layer of the $1^{\text {st }}$ iteration, were collected, and their UV-Vis spectroscopy shows that they were not good candidates for getting pure $(6,5)$ tubes because their UV-Vis spectrum showed the peak difference between $982 \mathrm{~nm}$ and $1031 \mathrm{~nm}$ was not as high was achieved the first layer of the $1^{\text {st }}$ iteration which meant it was easier to get pure $(6,5)$ tubes from the first layer of the centrifuge tubes than the second and the third layer of the centrifuge tube. Figure 3-6 and Figure 3-7 show UV-Vis graph of SWCNTs for the second and the third layer of the tube, respectively.All the solutions collected from the first layer of the first iteration were used for the second one, and the running conditions are the same as previously mentioned with the first iteration. Figure 3-6 shows the second iteration of SWCNTs. 


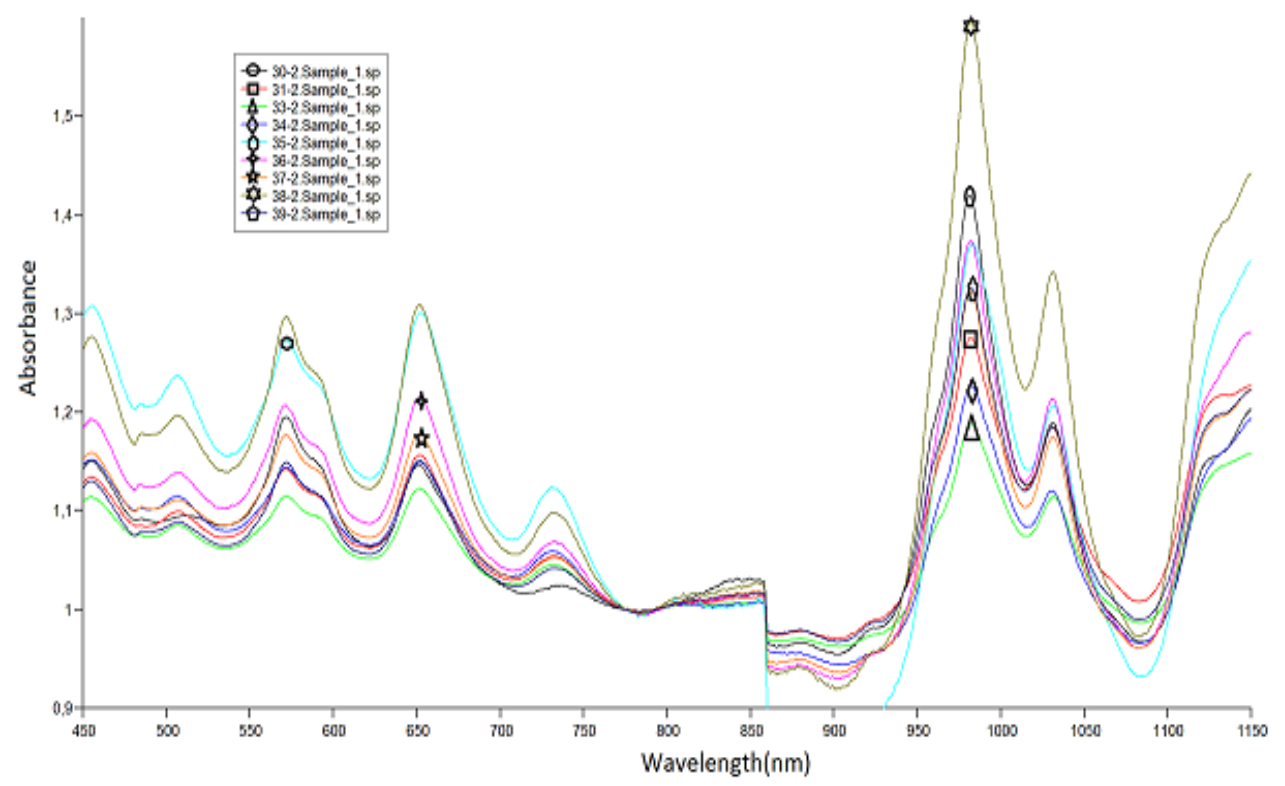

Figure 3-6The $1^{\text {st }}$ iteration of the second layer of the centrifuge tubes

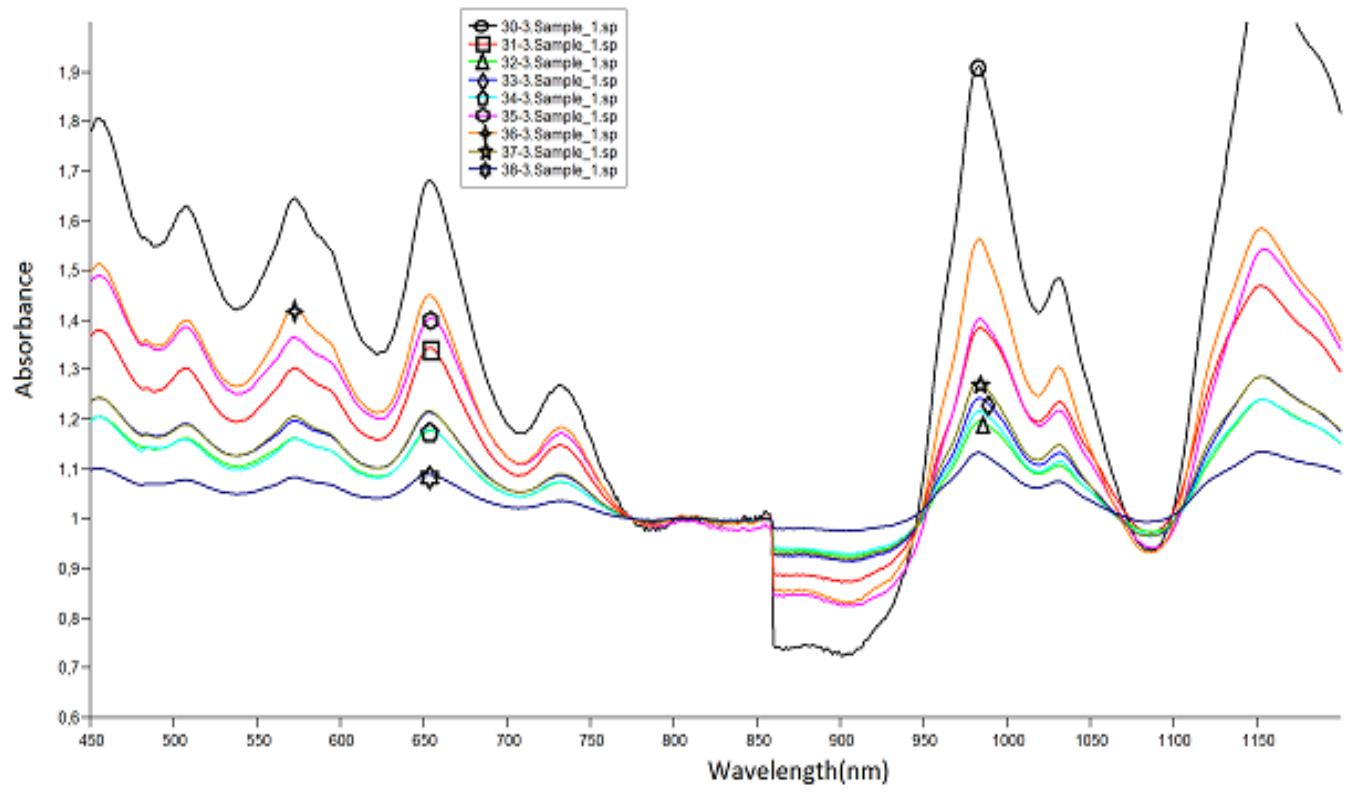

Figure 3-7The $1^{\text {st }}$ iteration of the second layer of the centrifuge tubes

After the $2^{\text {nd }}$ iteration of the $1^{\text {st }}$ layer, two distinct layers were observed, and peaks from $(6,5)$ tubes were observed stronger and $(7,5)$ peaks began to display a weakened. Figure 3-8 shows that centrifugation was performed with the top layer of the solution in order to get a pure $(6,5)$ tubes. Sample TA-1,XA-1 and XY-1 are the $2^{\text {nd }}$ iteration of the first layer samples, and they show a big intensity difference between $(6,5)$, and $(7,5)$ peaks. 


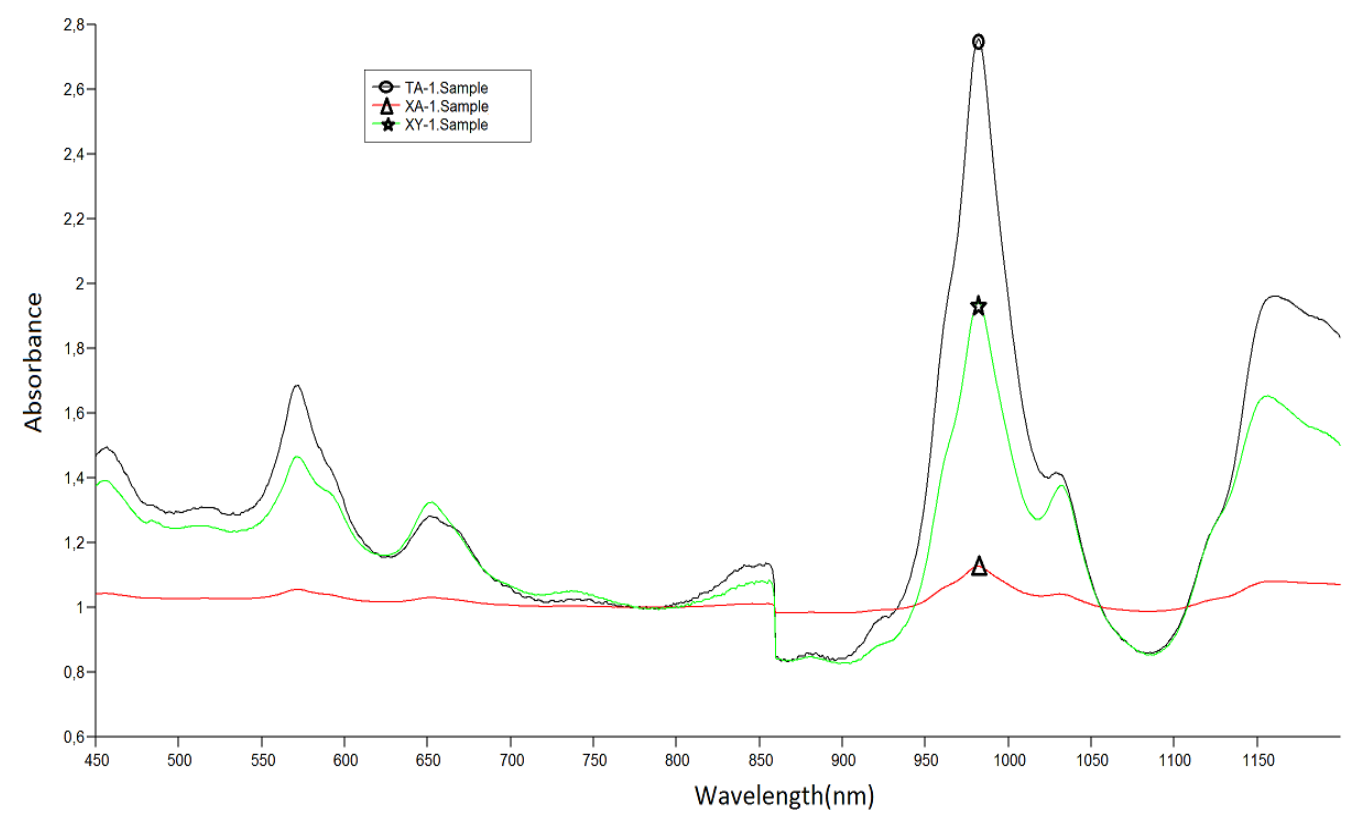

Figure 3-8UV-Vis graph of the first layer of the tubes with the second iteration

Figure 3-9 shows the second layer of the tubes with the second iteration. Second layer of the tubes with the second iteration shows that the difference between $(6,5)$ peak and $(7,5)$ peak is not as high as the first layer, so for the third iteration, the first layer is a good candidate in terms of getting pure $(6,5)$ tubes. Peak intensity difference is very low for the TA-2, XA-2 and XY-2 samples.

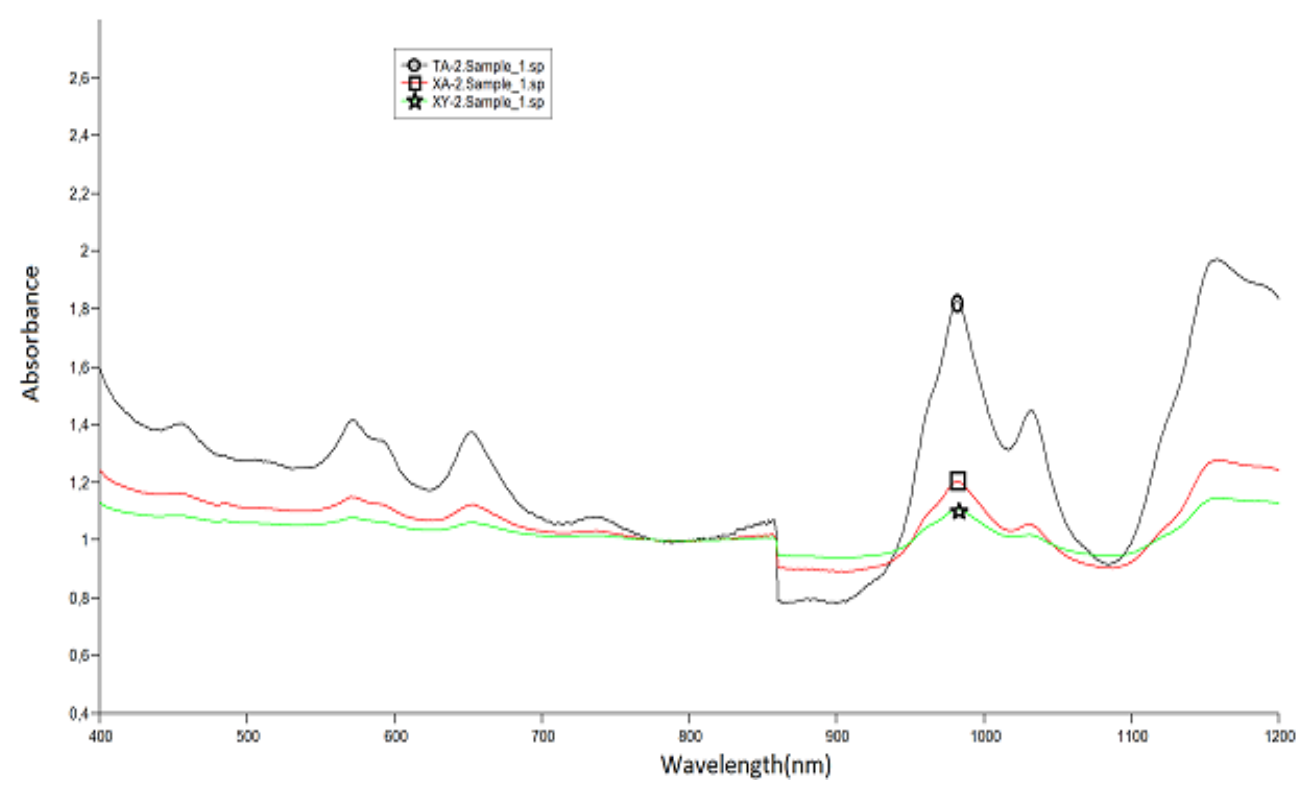

Figure 3-9UV-Vis graph of the second layer of the tubes with the second iteration 
Centrifugation was performed again with the same test conditions. Solutions from the second iteration were collected for the $3^{\text {rd }}$ run. Figure 3-10 shows that after the $3^{\text {rd }}$ iteration pure $(6,5)$ tubes can be achieved. . In the UV-Vis graph, there are two major peaks, one is around $571 \mathrm{~nm}$ another one is around $982 \mathrm{~nm}$.

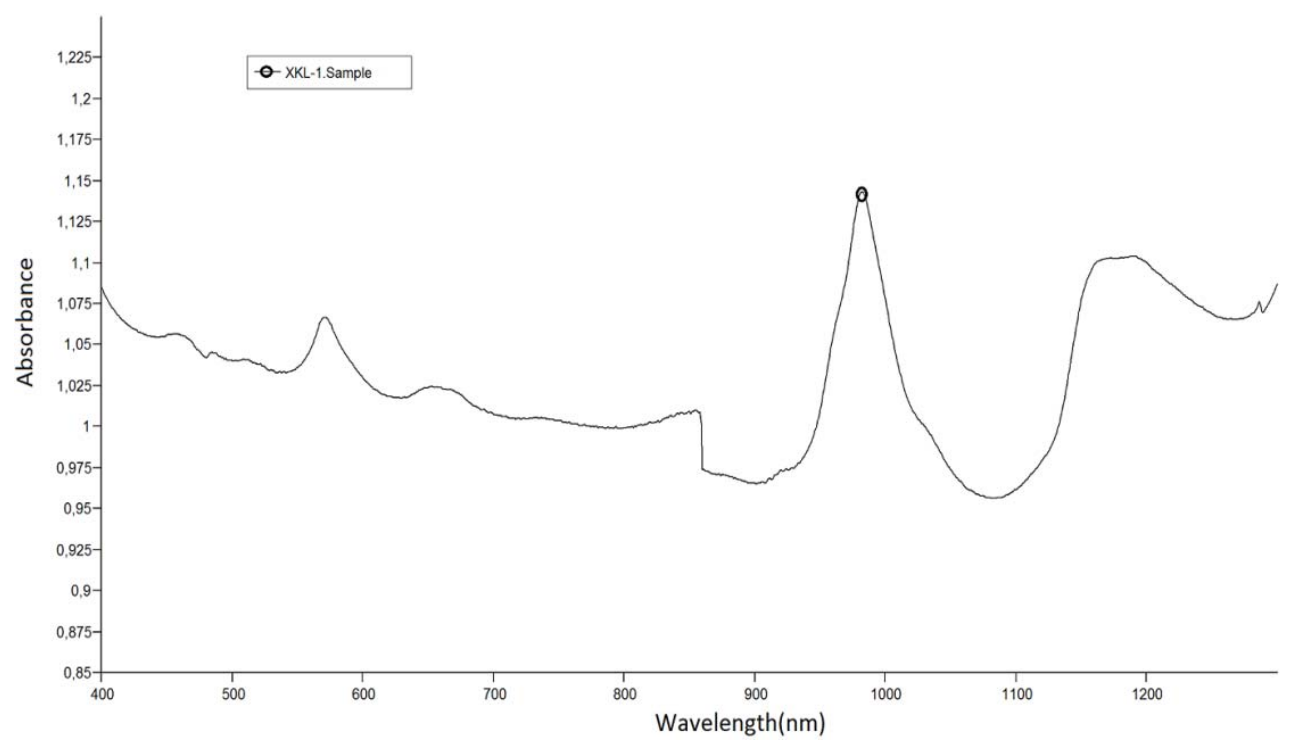

Figure 3-10UV-Vis graph of the first layer of the tubes after the third iteration

Figure 3-11 shows that the $3^{\text {rd }}$ iteration of the second layer and the third layer still has some $(7,5)$ species in it, so the $3^{\text {rd }}$ iteration the first layer is pure $(6,5)$ tubes.

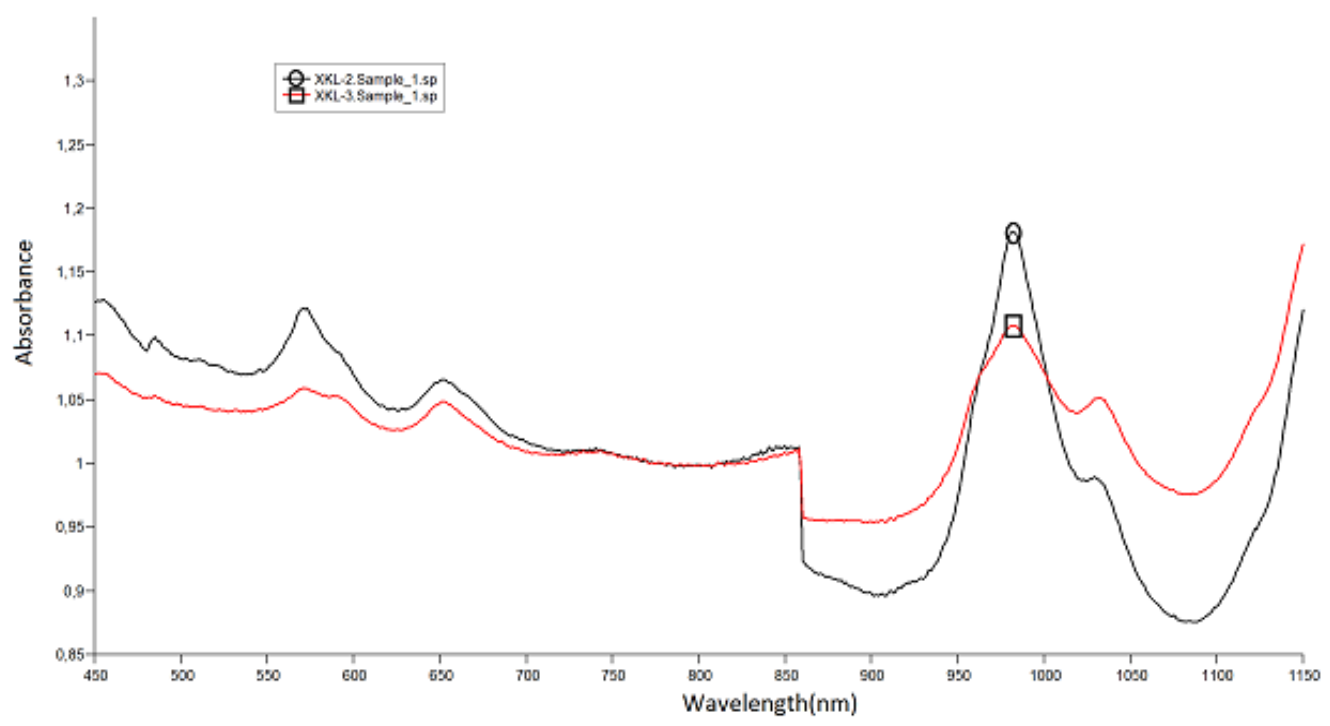

Figure $3-113^{\text {rd }}$ iteration second and the third layer of the tubes UV-Vis graph 
PerkinelmerRamanMicro 200 series $785 \mathrm{~nm}$ diode laser, with 4 seconds exposure time and number of exposure are set to 4 and 100x objective was used to characterize the nanotubes. Figure 3-12 shows the Raman spectroscopy of unsorted SWCNTs.

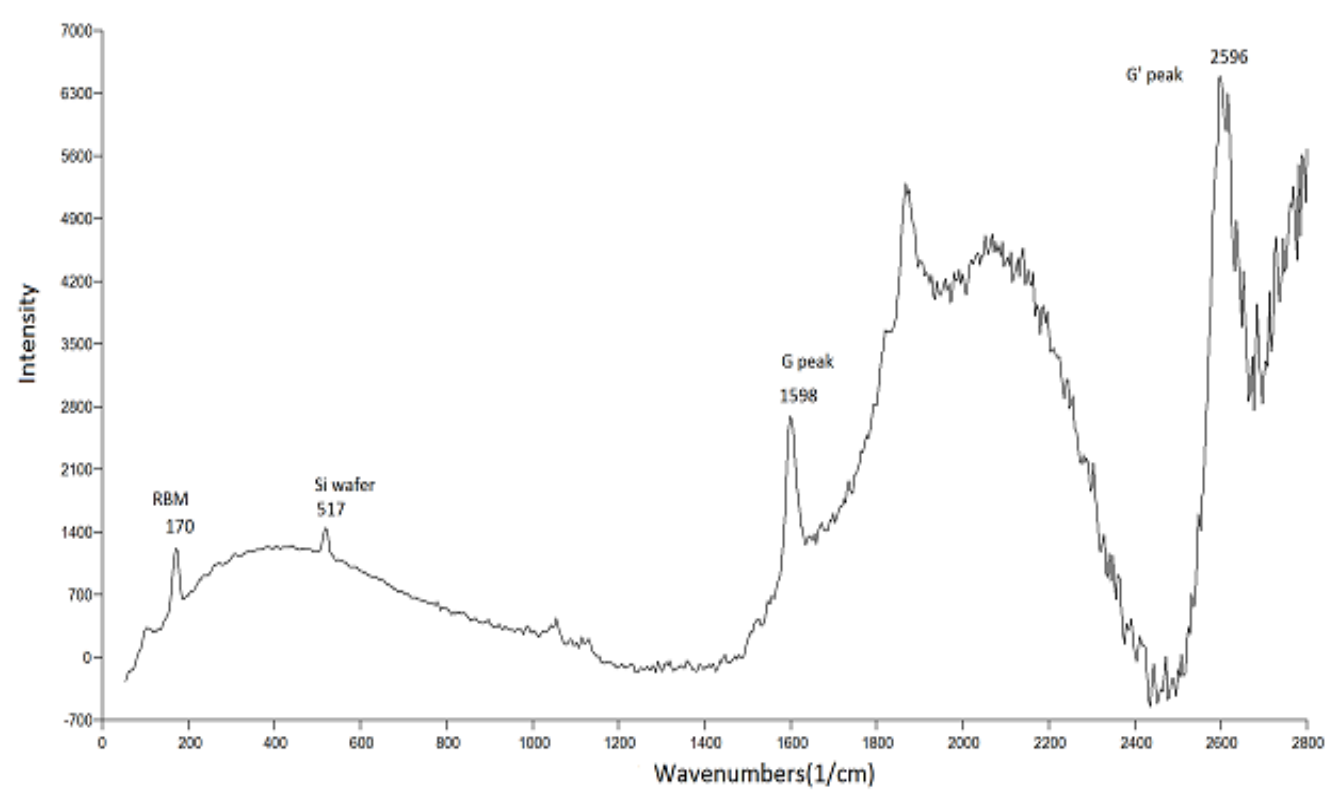

Figure 3-12Raman spectroscopy of unsorted SWCNTs

RBM peaks has useful information about the chirality of the SWCNTs. Figure 313shows that there are $235 \mathrm{~nm} 267 \mathrm{~nm}$ and 303nm peaks. According to UV-Vis studies pink layer shows $(6,5)$ tubes peak. $(6,5)$ tubes have to have $307 \mathrm{~nm}$ signal from the Raman graphs[52]. Moore et al. shows that using different surfactant might be the reason for different peaks at different locations like G peak and D peak, but using different surfactants are not going to change line positions of the radial breathing modes(RBMs)[53]. Using different production technique for the carbon nanotube results different line positions in the radial breathing mode[54], so this might be the reason for having RBM peak at $303 \mathrm{~nm}$. 


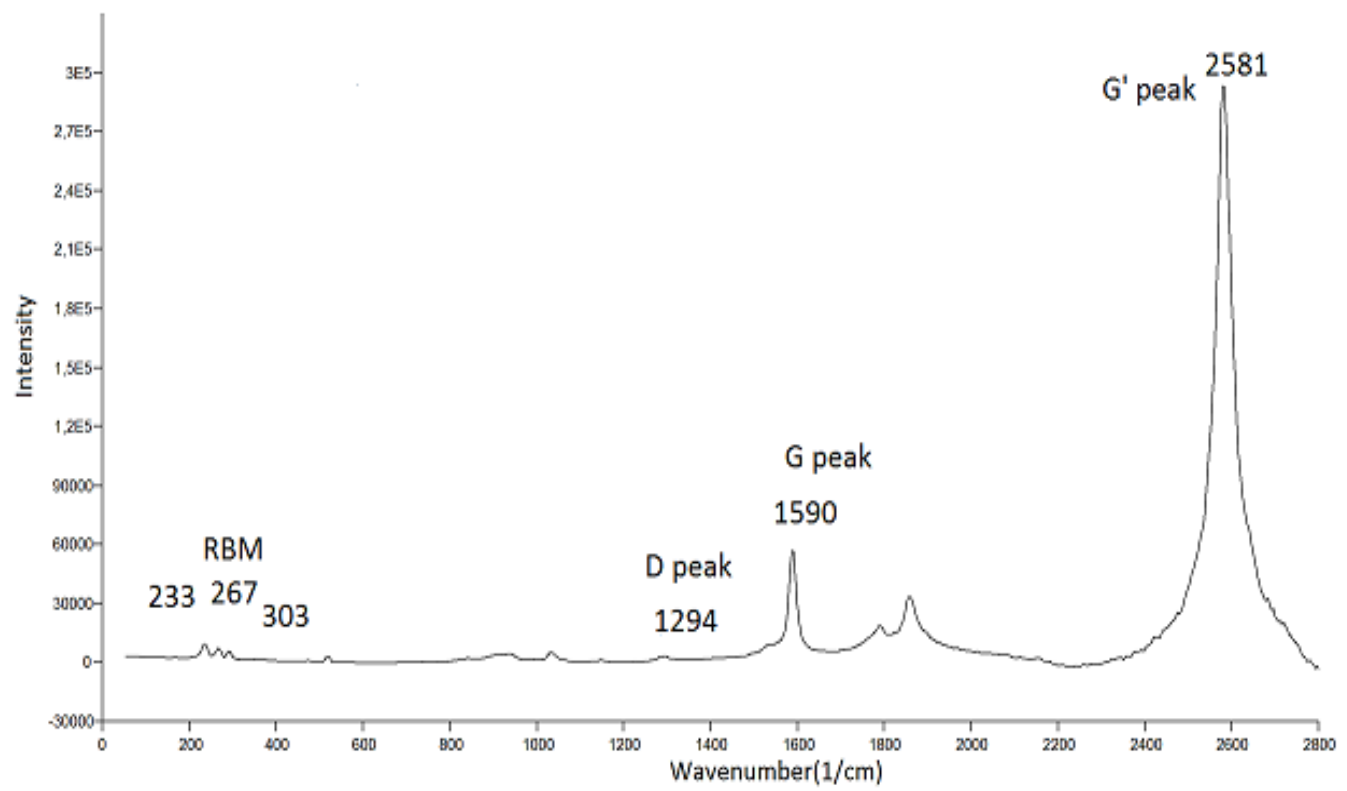

Figure 3-13Raman graph of pink layer

The calculation of RBM peaks location in Raman spectroscopyis different with the different production techniques. The constant values differs for different SWCNTS produced by different methods, and there is no braod study about ComoCAT type nanotubes and their RBM values[55], so according the findings CoMoCAT $(6,5)$ tube have a peak around $303 \mathrm{~nm}$.

\subsubsection{Green Layer $((6,5)+(7,5))$}

There was another layer observed in the centrifuge tube other than the pink $(6,5)$ layer, and this layer was a green in color. The same procedure was followed for this layer as with the pink layer, and UV-Vis spectroscopy and Raman spectroscopy characterization techniques performed on this layer, and for the UV-Vis spectrum graph showed the strong peaks around $982 \mathrm{~nm}$ and $1031 \mathrm{~nm}$ each observation. Figure 3-14 shows that green layer consists of two different species. The $982 \mathrm{~nm}$ peak indicate that there are $(6,5)$ species in this layer, and $1031 \mathrm{~nm}$ peak indicate that there is a $(7,5)$ species as well. 


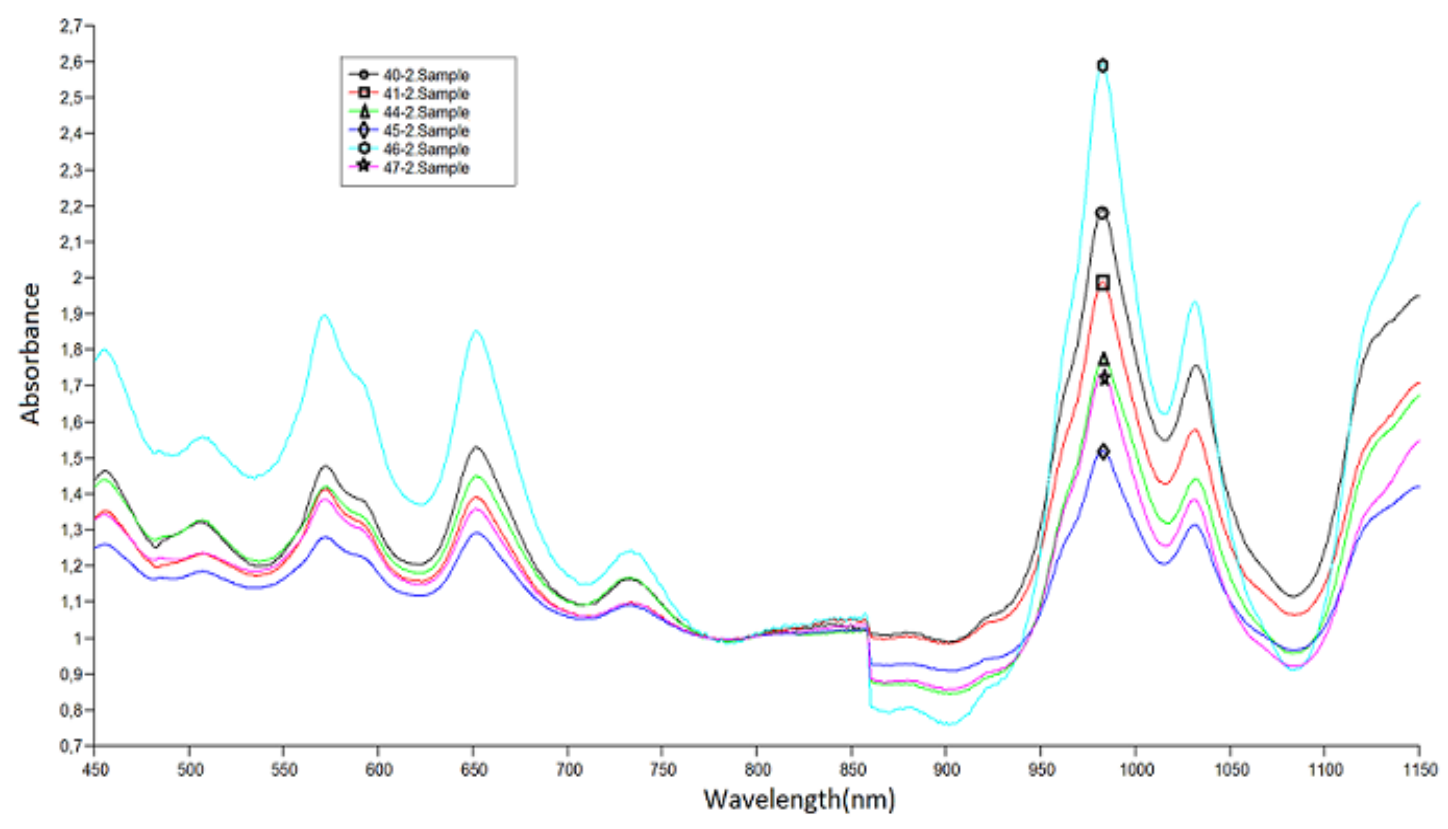

Figure 3-14UV-Vis graph of the green layer

Figure 3-15 shows Theraman study for the green layer. This layer has RBM peaks at $233 \mathrm{~nm}$ and $267 \mathrm{~nm}$.

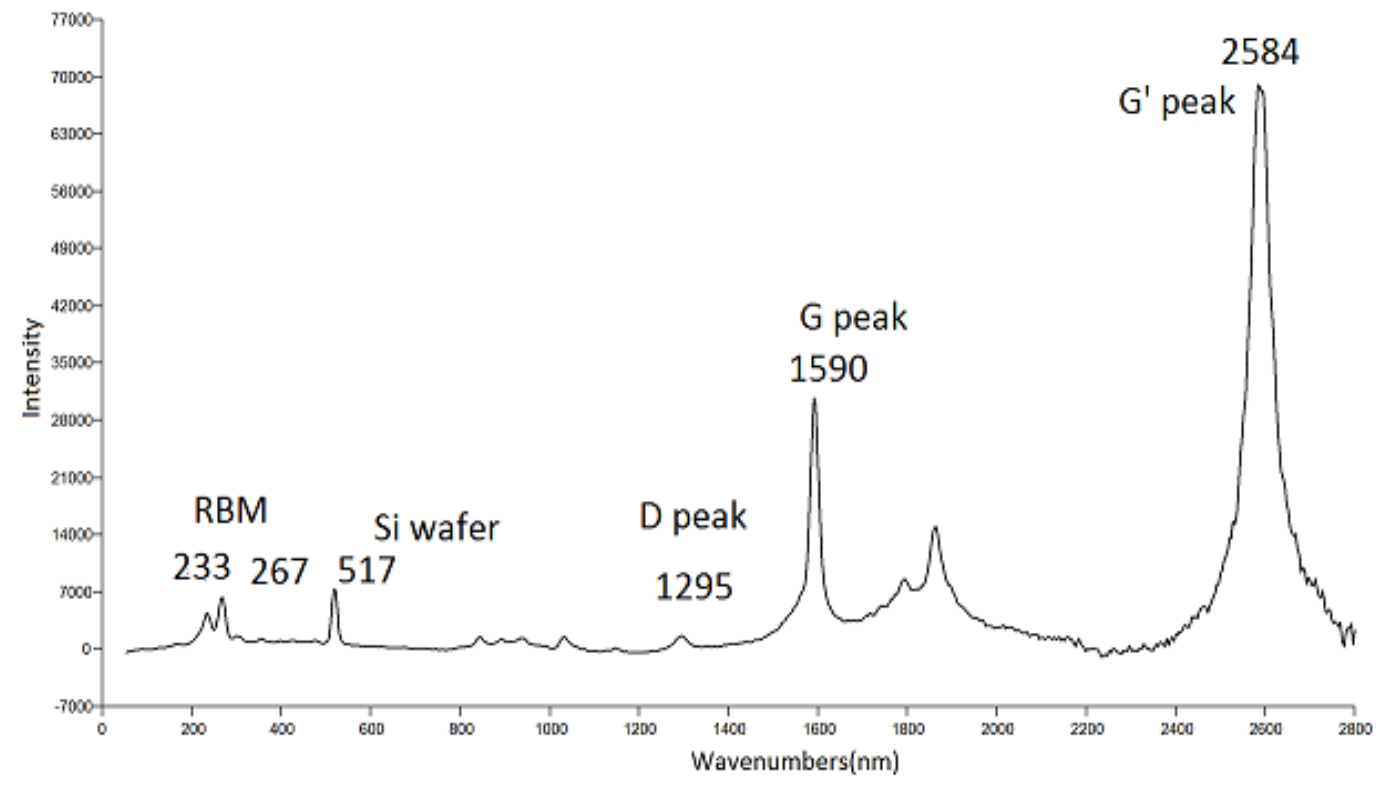

Figure 3-15Raman graph of green layer 
To visualize the presence of CNT's we used two different characterization techniques. First we use SEM image technique.

\subsection{Scanning Electron Microscopy}

A Semi-in-Lens FE: JSM-7600F supplied by JEOL was used to study the surface morphology of SWCNTs. The processed sample is placed on the specimen holder and the specimen stage to ensure uniform electrical conductivity of the assembly. Imageis captured at a vacuum of $9.6 \times 10^{-5} \mathrm{~Pa}$. The operating voltage was $15 \mathrm{kV}$ with resolution at $100 \mathrm{~nm}$ at 100,000 magnification.Figure 3-16 shows the SEM image of the SWCNTs on the cellulose ester membrane.

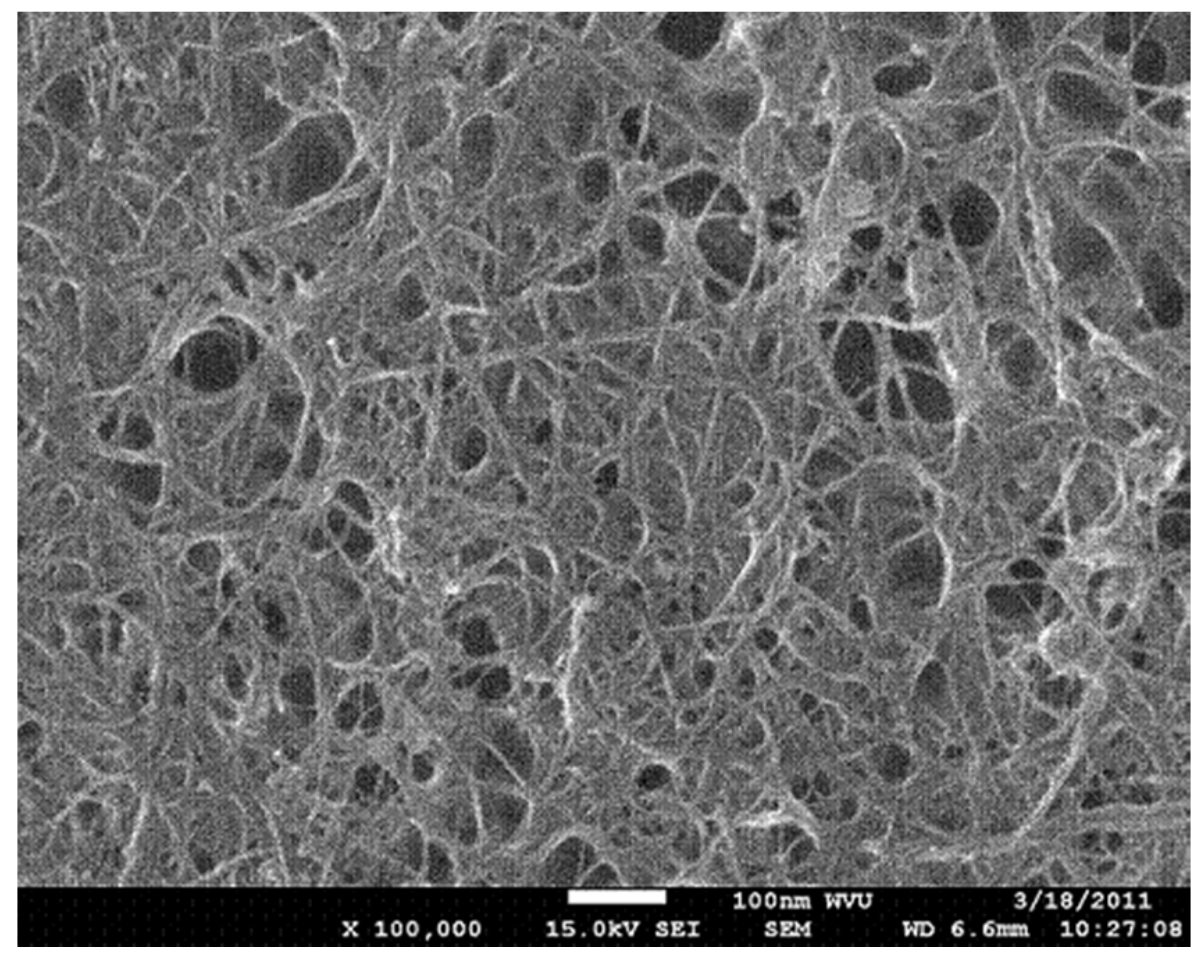

Figure 3-16SWCNTs on the cellulose membrane 


\subsection{Atomic Force Microscopy}

Second technique to visualize SWCNTs is the tapping mode AFM. For atomic imaging NanoscopeMultiMode Atomic Force Microscope that can characterize surface topography within range of $0.4 \mu$ laterally ( $\mathrm{x}$ and $\mathrm{y}$ axes) and $0.4 \mu$ vertically ( $\mathrm{z}$ axis) up to $200 \mu$ laterally and $10 \mu$ vertically with 16 bit resolution, and feedback control system managed by integral grain and proportional gain is used. PPP-NCL Cantilever specifications are $7 \pm 1 \mu \mathrm{m}$, length $225 \pm 10 \mu \mathrm{m}$, width $38 \pm 7.5 \mu \mathrm{m}$ and whose resonance frequency ranging between 146-236 kHz. Figure 3-17 shows the SWCNTs on the mica surface.

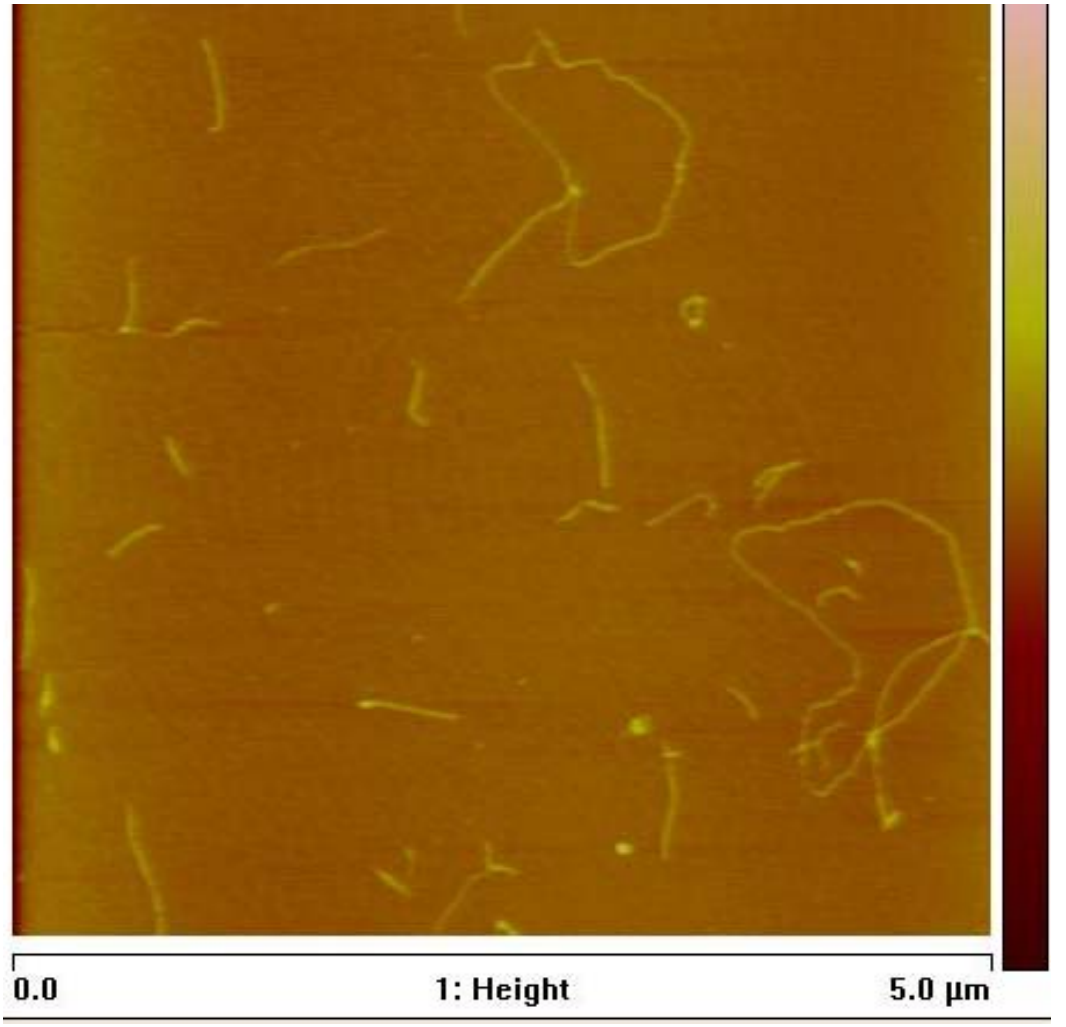

Figure 3-17SWCNTs on the mica surface 


\section{- CHAPTER 4 -}

\section{Electrically Conductive Thin Films}

Thin films of SWCNTs were deposited onto substrates to get properties that are not easy to achieve in the substrate alone. Thin films can be achieved by depositing multiple layers of different materials. There are several applications that thin films can be applied for production process. For optical purpose, it can be reflective/antireflective coatings, interference filters, memory discs and waveguides. For electrical applications, insulator, conductor semiconductor devices and piezoelectric drivers.For chemical applications, protection against oxidation or corrosion and gas/liquid sensors. For mechanical applications, tribological coatings, adhesion micromechanics. [56]

\subsection{Filtration Method}

There are several methods discovered by scientist to make nanotube films such as drop casting from solvents, spin coatings, dip casting etc. Carbon nanotubes film production is hard to control in terms of film thickness, homogeneity, uniformity, and flocculation between carbon atoms (Van der waals forces).It is difficult to uniformly coat SWNT thin films on substrates due to the aggregation of SWNTs[57].

In order to produce uniform films, vacuum filtration method was used in this project, and it involves vacuum filtering a dilute suspension of nanotubes in a solvent over a porous cellulose ester membrane. After the centrifugation, the solutions from different layer of the the centrifuge tube were vacuum filtered. As the solution goes through the membrane, nanotubes were trapped on the membrane surface, and forming interconnected network[58].

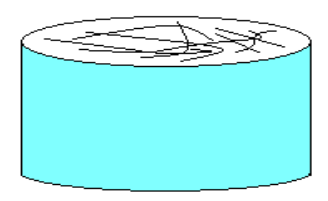

Figure 4-1Drawings of nanotube layer on a cellulose membrane 
Filtration method has several advantages. Homogenity of the films are consistent. Whenever SWCNTs accumulate on the membrane, thicker regions accumulation slowing down and thinner regions catch up the accumulation rate with the thicker regions. The film thickness is controllable by nanotube concentration and the volume of the suspension filtered[59]. A deposited SWCNT thin film on a cellulose ester membrane is shown below in Figure 4-2.

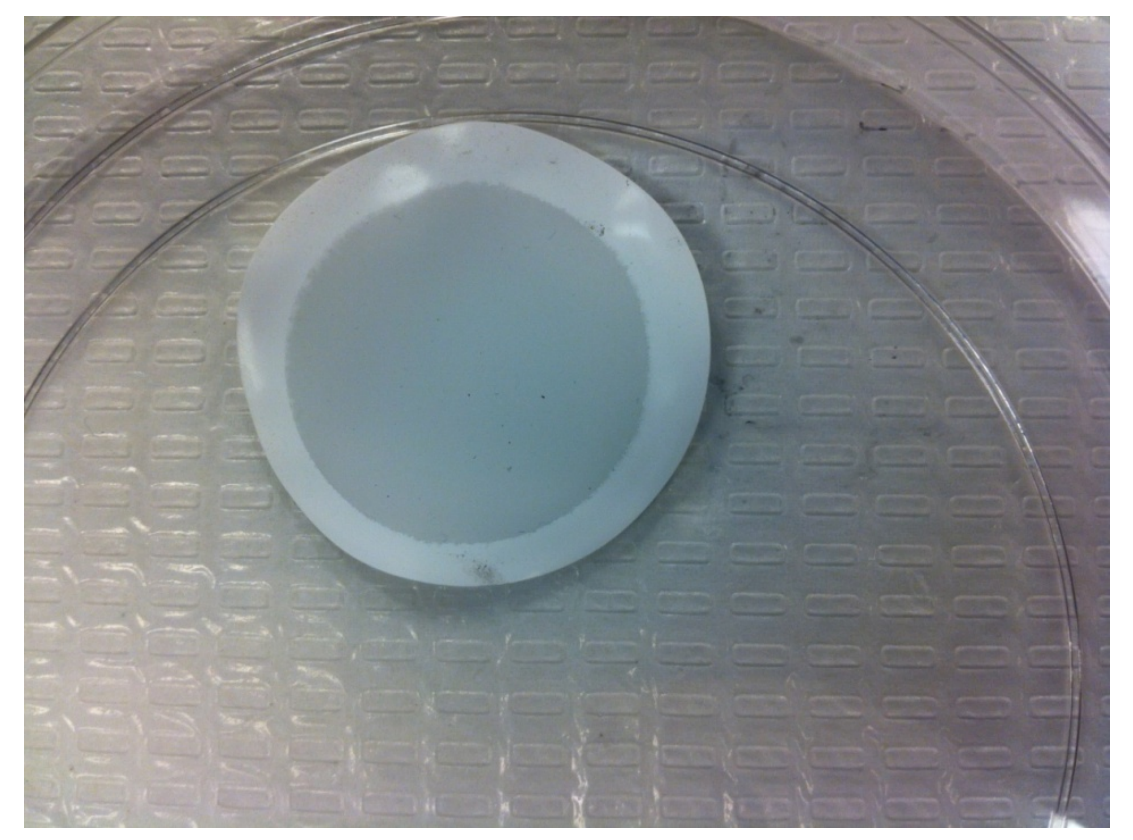

Figure 4-2Cellulose membrane and nanotube layer on the membrane

After getting carbon nanotubes on the cellulose membrane, the shadow mask can be designed for the electrode.

\subsection{Design of Shadow Mask}

For creating electrical network, cellulose ester membrane sputtered with $100 \mathrm{~nm}$ thick gold particles, and aluminum shadow mask used for this purpose. SolidWorks was used to design shadow mask. Figure 4-3 and Figure 4-4 show the design and dimensions of the mask in inches. 


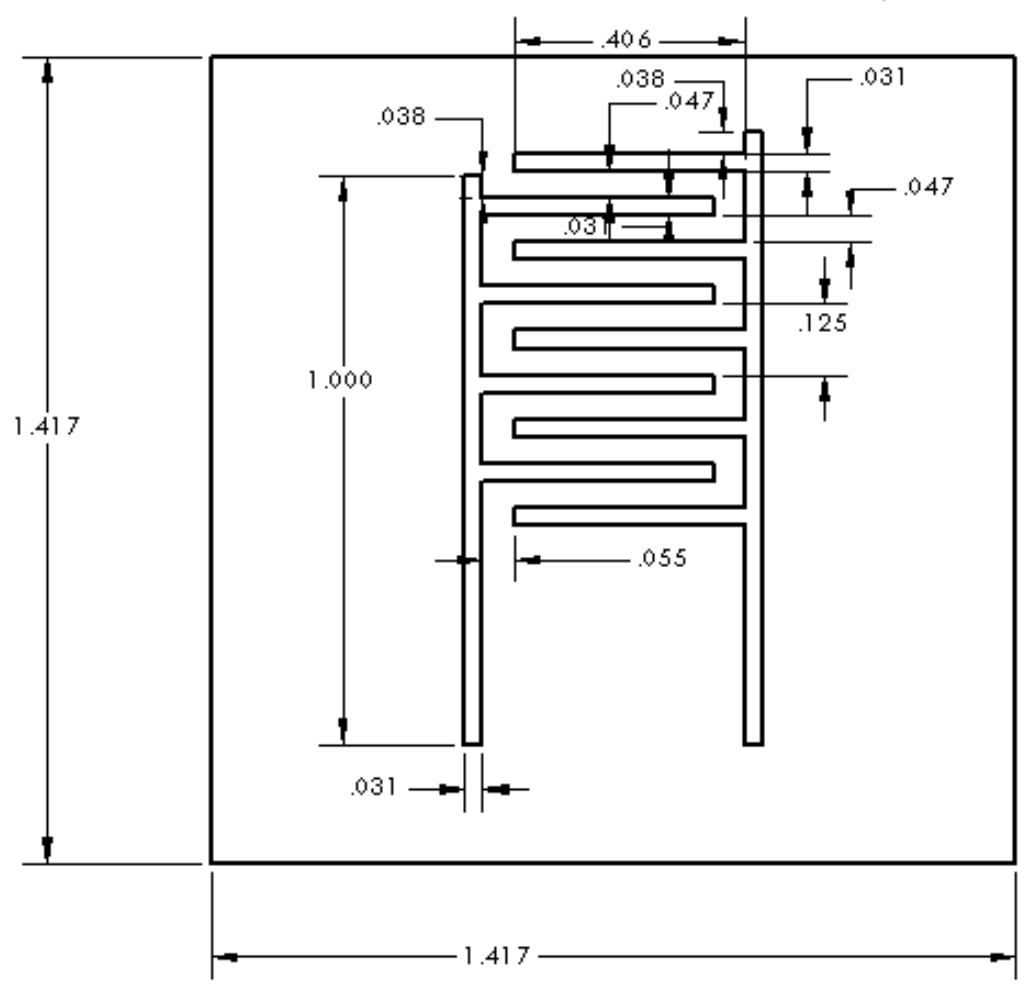

Figure 4-3Dimensions of the aluminum shadow mask

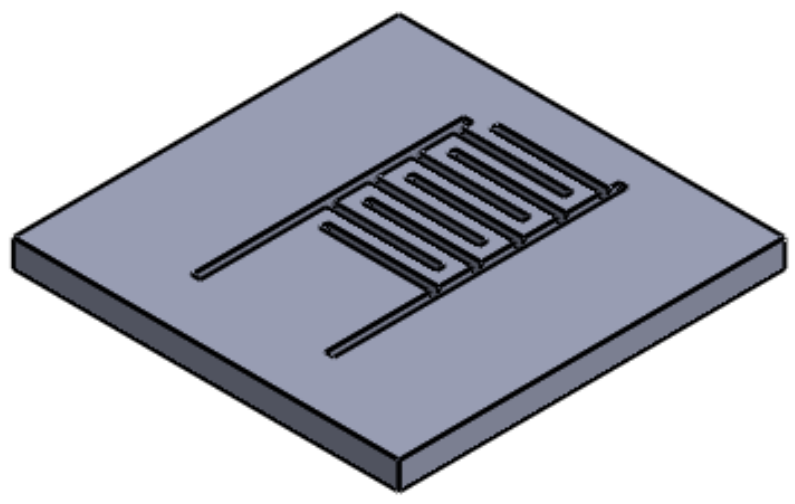

Figure 4-43D image of the shadow mask

Figure 4-5 shows the aluminum shadow masks and and Figure 4-6 shows the gold sputtered cellulose ester membranes with the help of shadow mask. 


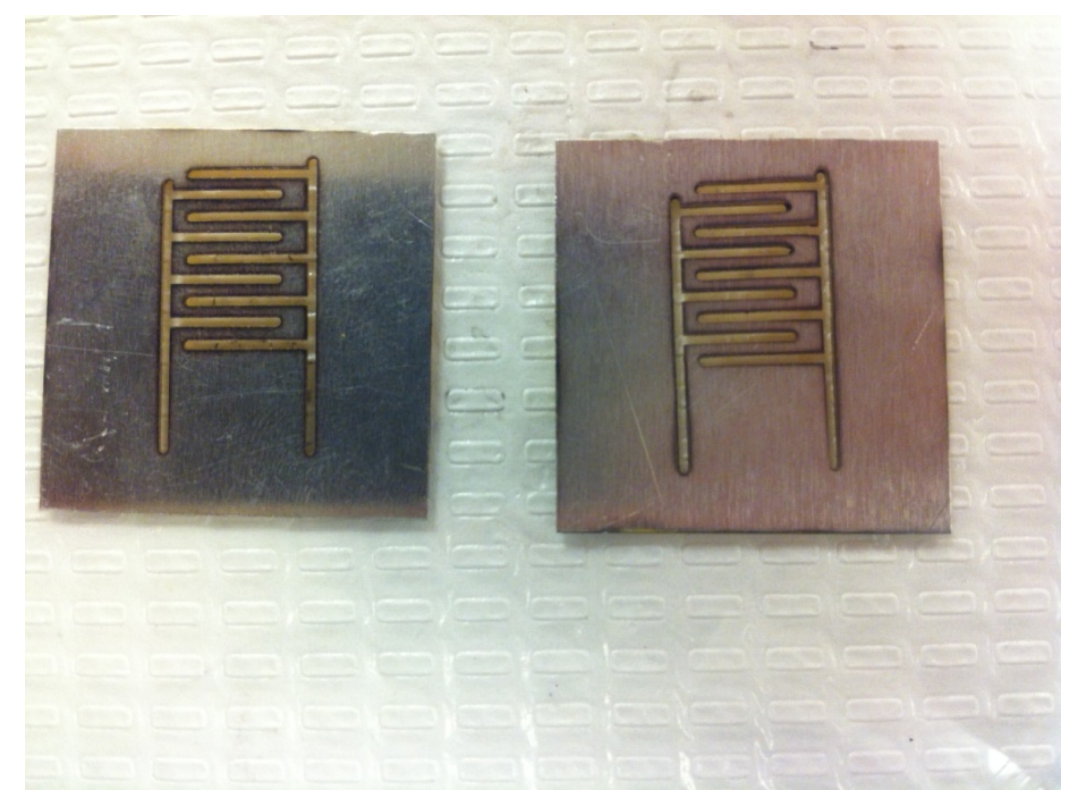

Figure 4-5Aluminum shadow masks

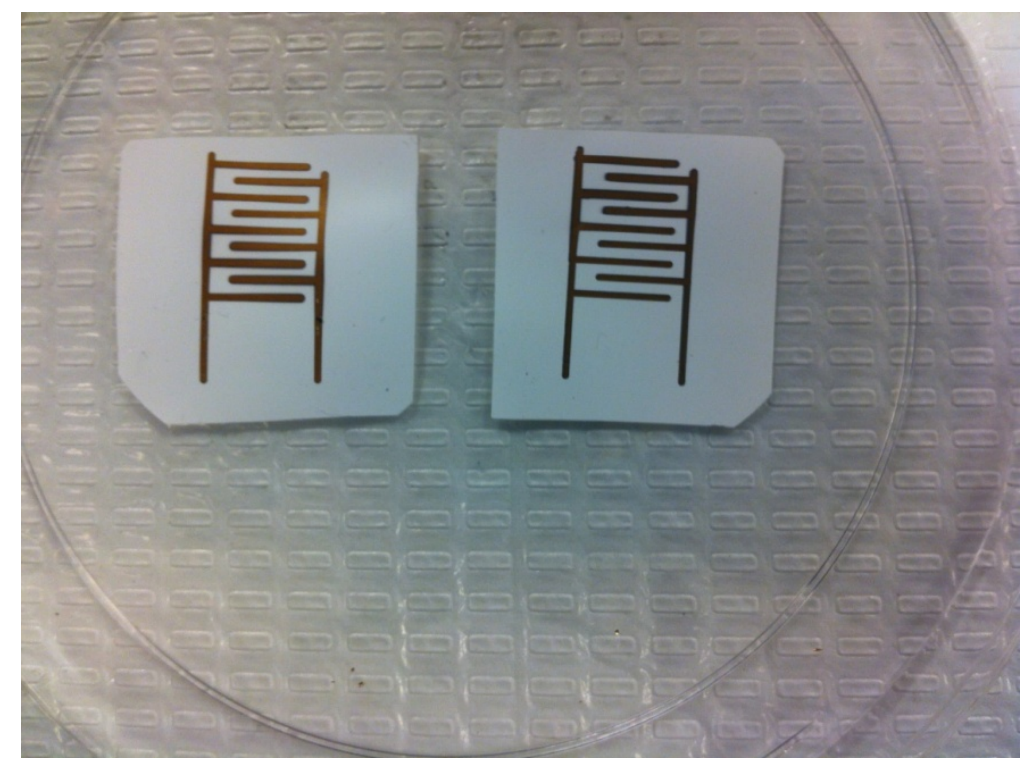

Figure 4-6Gold sputtered cellulose membranes

Figure 4-7 shows the gold sputtered SWCNTs network on the cellulose ester membrane. 


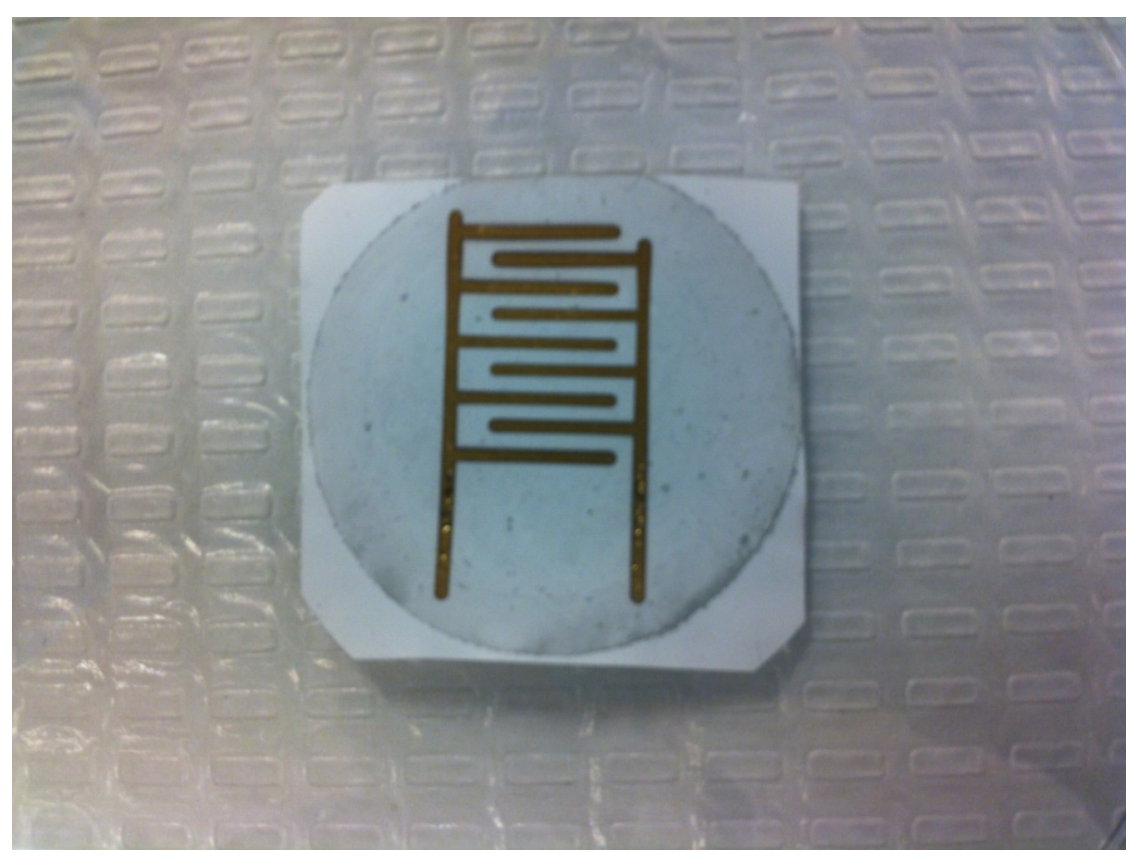

Figure 4-7Gold sputtered SWCNT network

A total of four pink and four green layered sampleswere prepared with different surface concentrations. Each sample sputtered with $100 \mathrm{~nm}$ thick gold film using aluminum shadow mask. After getting sputtered samples, each sample was analyzed with the Agilent 4294A precision impedance analyzer. The 16334A tweezer type test fixture is connected to the Agilent 4294A. Test fixture touched the surface of the gold sputtered cellulose membrane. Each time readings from the impedance analyzer at zero phase angle were taken. Table 4.1 and Table 4.2 shows the surface concentration values and impedance values.

Table 4.1 Green and pink samples surface concentration values.

\begin{tabular}{|l|l|c|c|c|c|}
\cline { 2 - 6 } \multicolumn{1}{c|}{} & & $\begin{array}{l}\text { Membrane } \\
\text { weight }(\mu \mathrm{g})\end{array}$ & $\begin{array}{l}\text { After } \\
\text { deposition } \\
\text { weight }(\mu \mathrm{g})\end{array}$ & $\begin{array}{l}\text { SWCNTs } \\
\text { weight. }(\mu \mathrm{g})\end{array}$ & $\begin{array}{l}\text { surface } \\
\text { concentration. } \\
\left(\mu \mathrm{g} / \mathrm{cm}^{\wedge} 2\right)\end{array}$ \\
\hline \multirow{3}{*}{ GREEN } & 1 & 90378 & 96320 & 5942 & 582 \\
\cline { 2 - 6 } & 2 & 91820 & 94540 & 2720 & 266 \\
\cline { 2 - 6 } & 3 & 92181 & 93973 & 1792 & 175 \\
\cline { 2 - 6 } & 4 & 92517 & 92892 & 375 & 36.72 \\
\hline PINK & 1 & 91274 & 94175 & 2901 & 284.13 \\
\cline { 2 - 7 } & 2 & 91053 & 95202 & 4149 & 406.36 \\
\cline { 2 - 7 } & 3 & 90631 & 97248 & 6616 & 648 \\
\cline { 2 - 6 } & 4 & 90972 & 100340 & 9368 & 917 \\
\hline
\end{tabular}


Table 4.2 Impedance values of different samples

\begin{tabular}{|l|r|l|r|r|}
\cline { 2 - 5 } \multicolumn{1}{c|}{} & & Frequency $(\mathrm{Hz})$ & Impedance(ohm) & phase angle(0) \\
\hline \multirow{4}{*}{ GREEN } & 1 & $40 \mathrm{~Hz}$ & $2.56 \mathrm{E}+02$ & $-2.21 \mathrm{E}-05$ \\
\cline { 2 - 5 } & 2 & $40 \mathrm{~Hz}$ & $1.08 \mathrm{E}+04$ & $-3.51 \mathrm{E}-03$ \\
\cline { 2 - 5 } & 3 & $40 \mathrm{~Hz}$ & $3.16 \mathrm{E}+04$ & $-1.54 \mathrm{E}-01$ \\
\cline { 2 - 5 } & 4 & $40 \mathrm{~Hz}$ & $3.05 \mathrm{E}+07$ & $-4.83 \mathrm{E}-01$ \\
\hline PINK & 1 & $40 \mathrm{~Hz}$ & $3.70 \mathrm{E}+07$ & $-4.18 \mathrm{E}-01$ \\
\cline { 2 - 5 } & 2 & $40 \mathrm{~Hz}$ & $2.07 \mathrm{E}+05$ & $-1.34 \mathrm{E}-02$ \\
\cline { 2 - 5 } & 3 & $40 \mathrm{~Hz}$ & $4.38 \mathrm{E}+03$ & $-1.31 \mathrm{E}-03$ \\
\cline { 2 - 5 } & 4 & $40 \mathrm{~Hz}$ & $7.65 \mathrm{E}+03$ & $-1.14 \mathrm{E}-02$ \\
\hline
\end{tabular}

Figure 4-8 shows how to use tweezer type test fixture.

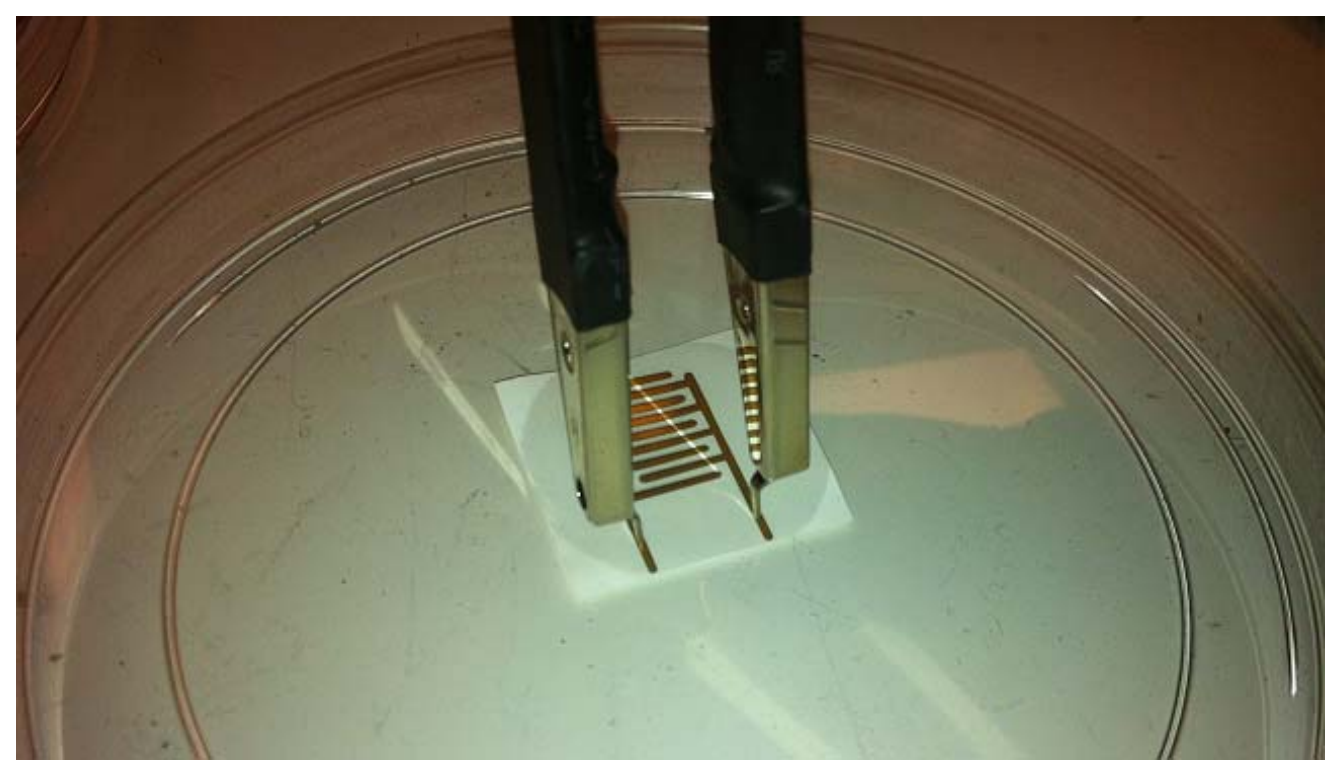

Figure 4-8Demonstration of the using tweezer type test fixture

The resistivity $(\rho)$ of the samples were calculated using the mask dimensions $(\mathrm{d})$, the impedance values $(Z)$ and the phase angle $(\theta)$ as shown in equation 1

$\rho=\left|z_{S}\right| \cos \theta\left(\left(\# d_{3} \cdot\left(\frac{d_{3}}{d_{4}}\right)\right)+\left(\# d_{2} \cdot\left(\frac{d_{2}}{d_{1}}\right)\right)\right)[60]$

The conductivity $(\sigma)$ was calculated using equation(2)

$\sigma=1 / \rho$ 
$\# d_{3}$ : number of fingers

$\# d_{2}$ : number of spaces between fingers

$d_{1}: 0.054687$ in

$d_{2}: 0.03125$ in

$d_{3}: 0.40628$ in

$d_{4}: 0.04687$ in

Figure 4-9 shows the dimensions of the design, and Table 4.3. shows the surface concentration values of the samples and conductivity values.

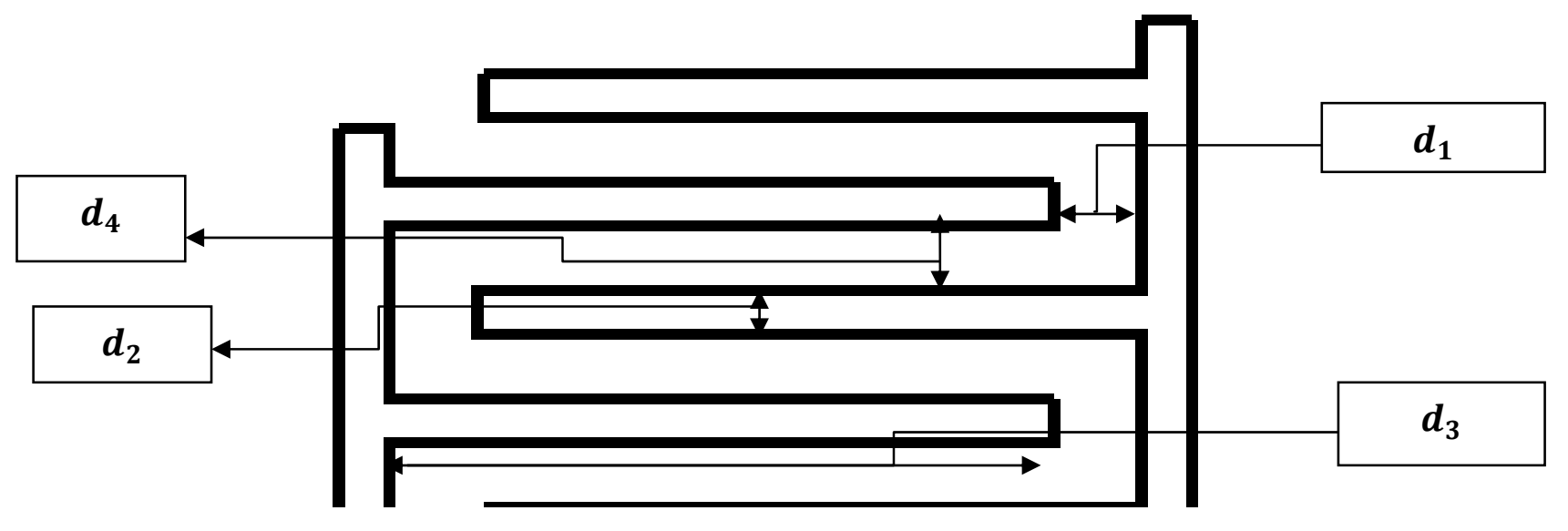

Figure 4-9Dimensions of the design

Table 4.3 shows the SWCNT thin film samples calculated conductivity and surface concentration values.

Table 4.3.Green vs pink layer conductivity and surface conc. values

\begin{tabular}{|l|l|l|l|l|c|c|}
\cline { 2 - 7 } \multicolumn{1}{c|}{} & & $\begin{array}{l}\text { Membrane } \\
\text { weight. }(\mu \mathrm{g})\end{array}$ & $\begin{array}{l}\text { After } \\
\text { deposition } \\
\text { weight }(\mu \mathrm{g})\end{array}$ & $\begin{array}{l}\text { SWCNT } \\
\text { weight. } \mu \mathrm{g})\end{array}$ & $\begin{array}{l}\text { surface } \\
\text { concentration } \\
\left(\mu \mathrm{g} / \mathrm{cm}^{\wedge} 2\right)\end{array}$ & $\begin{array}{l}\text { Conductivity } \\
\text { (S square) }\end{array}$ \\
\hline \multirow{3}{*}{ GREEN } & 1 & 90378 & 96320 & 5942 & 582 & $4.72 \mathrm{E}-05$ \\
\cline { 2 - 7 } & 2 & 91820 & 94540 & 2720 & 266 & $1.12 \mathrm{E}-06$ \\
\cline { 2 - 7 } & 3 & 92181 & 93973 & 1792 & 175 & $3.83 \mathrm{E}-07$ \\
\hline PINK & 4 & 92517 & 92892 & 375 & 36.72 & $3.97 \mathrm{E}-10$ \\
\cline { 2 - 7 } & 1 & 91274 & 94175 & 2901 & 284.13 & $3.27 \mathrm{E}-10$ \\
\cline { 2 - 7 } & 2 & 91053 & 95202 & 4149 & 406.36 & $5.84 \mathrm{E}-08$ \\
\cline { 2 - 7 } & 3 & 90631 & 97248 & 6616 & 648 & $2.76 \mathrm{E}-06$ \\
\hline
\end{tabular}


Table 4.3.shows that green layers conductivity value is higher than the pink layer conductivity value at very close surface concentration. We can conclude that the green layer which is mixture of $(6,5)$ and $(7,5)$ tubes has a higher conductivity than the pink layer which is only has $(6,5)$ tubes, so green layer is a good candicate for the applications which requires high conductivity.

Transparent and conducting single-wall carbon nanotube thin films transport mechanism can be analyzed in the framework of the percolation theory[61].Percolation theory has a significant importance SWCNT based electrically conductive networks. Reduction of the percolation threshold is very important because adding less SWCNT on the network is going to reduce the cost of the system. There have been numerical and analytical models proposed about the percolation theory, and scientists found out that interactions between SWCNTs have an important role for percolation threshold[62].Figure 4-10 shows a two dimensional percolation of randomly dispersed high aspect-ratio particles.

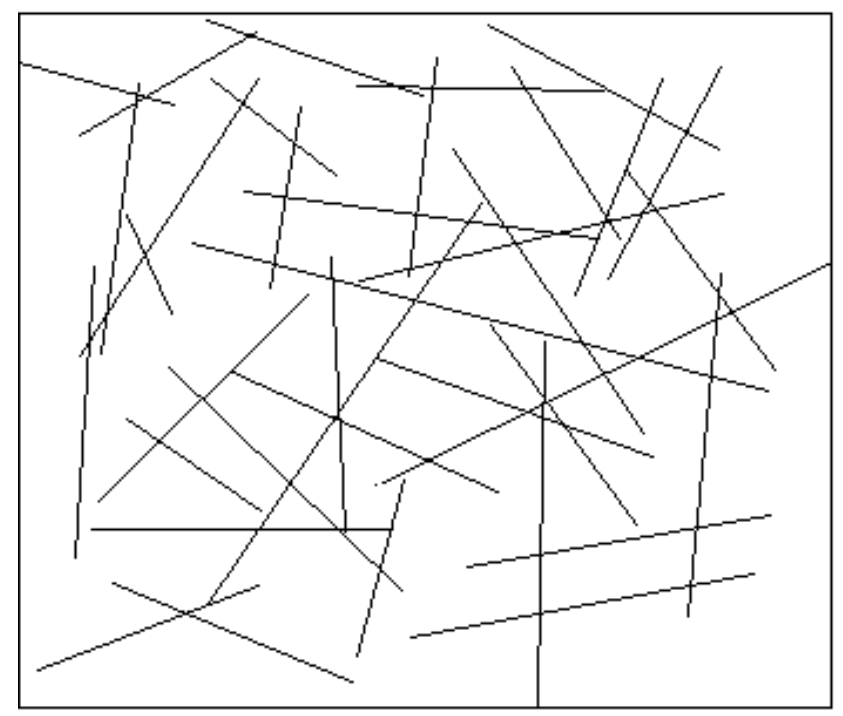

Figure 4-10A two-dimensional schematic of the percolation of randomlydispersed high aspect-ratio particles.

Electrical percolation is related to geometrical percolation, and geometrical percolation is the formation of an infinite network ofparticles that are connected[63]. 
The small size and the high aspect ratio of CNT allow the electrical percolation at low loadings, and having conductive material with very low loadings is important for many electrical applications[64]. Figure 4-11 shows the relation between conductivity and surface concentration of the thin film samples.

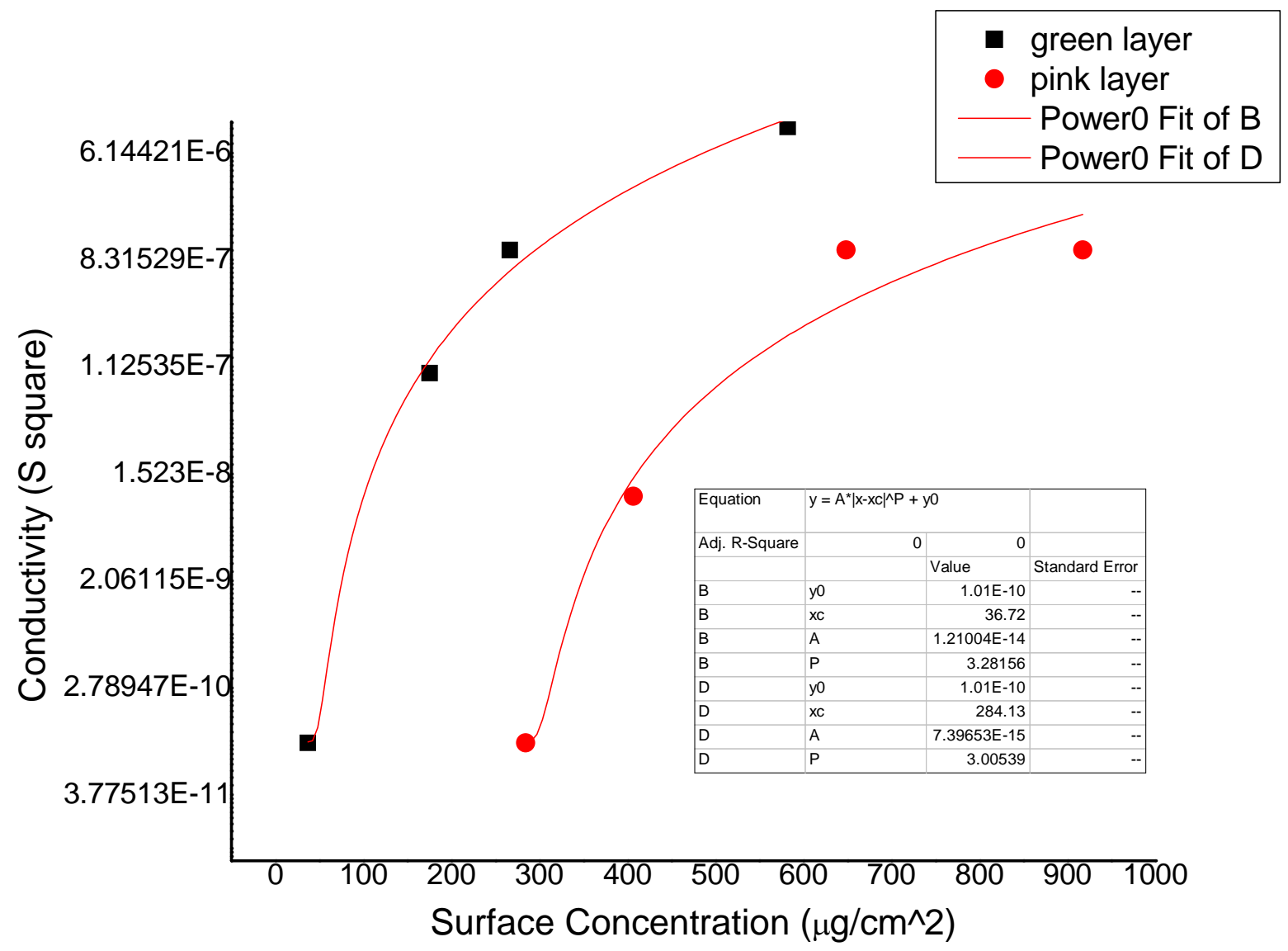

Figure 4-11Conductivity vs surface Concentration

The variation of electrical conductivity of the network shows a classical S curve behavior. Figure 4-11shows the typical S curve behavior. The critical point of SWCNT loading on the systems which result in a drastic change in the conductivity referred to as the percolation threshold. Percolation threshold ranges between 75 and $150 \mu \mathrm{g} / \mathrm{cm}^{2}$ surface concentration for the green layer, and 300 and $450 \mu \mathrm{g} / \mathrm{cm}^{2}$ for the pink layer. At the post percolation threshold levels of SWCNT loading electrical conductivity shows very weak changes in terms of volume fraction of SWCNT. 


\section{- CHAPTER 5 -}

\section{Conclusion and future directions}

Sorting process of single walled carbon nanotubes is an essential process in order to get high benefit from the single walled carbon nanotube network. Length sortingand type sorting processes have been done by many people. Chirality sorting has been accomplished by groups[36, 41], and in this projectComoCAT type carbon nanotubes were used in order to sort them by their chirality.First, SWCNT powder was sonicated with the SC solution and then several solutions were prepared with the iodixanol and deionized water. Sonicated SWCNT were run in the ultracentrifuge in order to remove catalyst and impurities. Different solutions with the different percentages were prepared. Ultracentrifugation was performed at $61.000 \mathrm{rpm}$ at $22^{\circ} \mathrm{C}$. The first layer of the SWCNT after centrifugation was collected, and those solutions were run again in order to isolate $(6,5)$ tubes. After the third iteration, pure $(6,5)$ SWCNTs were achieved. UV-Vis data shows that good isolation of $(6,5)$ tubes were attained. A secondary layer with a green color was also investigated. This layer was a mixture of $(6,5)$ and $(7,5)$ species. After getting all the sorted tubes, solutions were deposited on the cellulose ester membrane. A mask was created with the aluminum plate, of an electrode use on the surface of the deposited SWCNT thin films. The electrode deposited had a thickness of $100 \mathrm{~nm}$ and was sputtered from a pure gold target. Impedance measurement performed werewith the Agilent impedance analyzer. Conductivity vs surface concentration relationship shows typical S curve behavior. At the certain point, conductivity does not change with the concentration. A future direction is to extract the percolation threshold quantitatively from the curve by fitting the data using nonlinear fitting to the GEM model. 


\section{References}

[1] S. Iijima, "Helical Microtubules of Graphitic Carbon," Nature, vol. 354, pp. 5658, Nov 71991.

[2] Y. M. Lin, et al., "Electrical transport and $1 / \mathrm{f}$ noise in semiconducting carbon nanotubes," Physica E: Low-dimensional Systems and Nanostructures, vol. 37, pp. 72-77, 2007.

[3] E. S. Snow, et al., "1/f noise in single-walled carbon nanotube devices," Applied Physics Letters, vol. 85, pp. 4172-4174, Nov 12004.

[4] J. P. Salvetat-Delmotte and A. Rubio, "Mechanical properties of carbon nanotubes: a fiber digest for beginners," Carbon, vol. 40, pp. 1729-1734, 2002.

[5] S. Sinha, et al., "Off-axis thermal properties of carbon nanotube films," Journal of Nanoparticle Research, vol. 7, pp. 651-657, Dec 2005.

[6] S. Hong and S. Myung, "Nanotube electronics - A flexible approach to mobility," Nature Nanotechnology, vol. 2, pp. 207-208, Apr 2007.

[7] M. C. Hersam, "Progress towards monodisperse single-walled carbon nanotubes," Nature Nanotechnology, vol. 3, pp. 387-394, Jul 2008.

[8] R. Saito, et al., Physical properties of carbon nanotubes. London: Imperial College Press, 1998.

[9] S. Campidelli, et al., "Separation of metallic and semiconducting single-walled carbon nanotubes via covalent functionatization," Small, vol. 3, pp. 1672-1676, Oct 2007.

[10] P. Ajayan and O. Zhou, "Applications of carbon nanotubes," Carbon Nanotubes, pp. 391-425, 2001.

[11] N. Komatsu and F. Wang, "A Comprehensive Review on Separation Methods and Techniques for Single-Walled Carbon Nanotubes," Materials, vol. 3, pp. 38183844, Jul 2010.

[12] R. H. Baughman, et al., "Carbon nanotubes--the route toward applications," Science, vol. 297, p. 787, 2002.

[13] Y. Zhang, et al., "Sorting out semiconducting single-walled carbon nanotube arrays by preferential destruction of metallic tubes using xenon-lamp irradiation," The Journal of Physical Chemistry C, vol. 112, pp. 3849-3856, 2008.

[14] J. M. Bonard, et al., "Carbon nanotube films as electron field emitters," Carbon, vol. 40, pp. 1715-1728, 2002.

[15] J. H. Hafner, et al., "Structural and functional imaging with carbon nanotube AFM probes," Progress in Biophysics \& Molecular Biology, vol. 77, pp. 73-110, 2001 .

[16] S. M. Lee and Y. H. Lee, "Hydrogen storage in single-walled carbon nanotubes," Applied Physics Letters, vol. 76, pp. 2877-2879, May 152000.

[17] P. Serp, et al., "Carbon nanotubes and nanofibers in catalysis," Applied Catalysis a-General, vol. 253, pp. 337-358, Oct 282003.

[18] D. Kim, et al., "Improved Thermoelectric Behavior of Nanotube-Filled Polymer Composites with Poly(3,4-ethylenedioxythiophene) Poly(styrenesulfonate)," Acs Nano, vol. 4, pp. 513-523, Jan 2010. 
[19] M. C. Hermant, et al., "Probing the Cooperative Nature of the Conductive Components in Polystyrene/Poly(3,4-ethylenedioxythiophene): Poly(styrene sulfonate)-Single-Walled Carbon Nanotube Composites," Acs Nano, vol. 4, pp. 2242-2248, Apr 2010.

[20] M. Glerup, et al., "Synthesis of N-doped SWNT using the arc-discharge procedure," Chemical Physics Letters, vol. 387, pp. 193-197, Mar 212004.

[21] W. K. Maser, et al., "Production of high-density single-walled nanotube material by a simple laser-ablation method," Chemical Physics Letters, vol. 292, pp. 587593, Aug 141998.

[22] X. Devaux and M. Vergnat, "On the low-temperature synthesis of SWCNTs by thermal CVD," Physica E-Low-Dimensional Systems \& Nanostructures, vol. 40, pp. 2268-2271, May 2008.

[23] R. Krupke and F. Hennrich, "Separation techniques for carbon nanotubes," Advanced Engineering Materials, vol. 7, pp. 111-116, Mar 2005.

[24] R. Krupke, et al., "Separation of metallic from semiconducting single-walled carbon nanotubes," Science, vol. 301, pp. 344-347, Jul 182003.

[25] S. K. Doorn, et al., "High resolution capillary electrophoresis of carbon nanotubes," Journal of the American Chemical Society, vol. 124, pp. 3169-3174, Mar 272002.

[26] S. K. Doorn, et al., "Capillary electrophoresis separations of bundled and individual carbon nanotubes," Journal of Physical Chemistry B, vol. 107, pp. 6063-6069, Jun 262003.

[27] X. Y. Huang, et al., "High-resolution length sorting and purification of DNAwrapped carbon nanotubes by size-exclusion chromatography," Analytical Chemistry, vol. 77, pp. 6225-6228, Oct 12005.

[28] M. Zheng, et al., "DNA-assisted dispersion and separation of carbon nanotubes," Nature Materials, vol. 2, pp. 338-342, May 2003.

[29] M. Zheng, et al., "Structure-based carbon nanotube sorting by sequencedependent DNA assembly," Science, vol. 302, pp. 1545-1548, Nov 282003.

[30] X. M. Tu and M. Zheng, "A DNA-Based Approach to the Carbon Nanotube Sorting Problem," Nano Research, vol. 1, pp. 185-194, Sep 2008.

[31] D. Chattopadhyay, et al., "A route for bulk separation of semiconducting from metallic single-wall carbon nanotubes," Journal of the American Chemical Society, vol. 125, pp. 3370-3375, Mar 192003.

[32] J. Y. Hwang, et al., "Polymer structure and solvent effects on the selective dispersion of single-walled carbon nanotubes," Journal of the American Chemical Society, vol. 130, pp. 3543-3553, Mar 192008.

[33] F. M. Chen, et al., "Toward the extraction of single species of sinale-walled carbon nanotubes using fluorene-based polymers," Nano Letters, vol. 7, pp. 30133017, Oct 2007.

[34] M. Yudasaka, et al., "Diameter-selective removal of single-wall carbon nanotubes through light-assisted oxidation," Chemical Physics Letters, vol. 374, pp. 132136, Jun 42003.

[35] Y. Miyata, et al., "Chirality-dependent combustion of single-walled carbon nanotubes," Journal of Physical Chemistry C, vol. 111, pp. 9671-9677, Jul 12 2007. 
[36] M. S. Arnold, et al., "Sorting carbon nanotubes by electronic structure using density differentiation," Nature Nanotechnology, vol. 1, pp. 60-65, Oct 2006.

[37] K. Yanagi, et al., "Separations of Metallic and Semiconducting Carbon Nanotubes by Using Sucrose as a Gradient Medium," Journal of Physical Chemistry C, vol. 112, pp. 18889-18894, Dec 42008.

[38] J. A. Fagan, et al., "Carbon Nanotubes: Measuring Dispersion and Length," Advanced Materials, vol. 23, pp. 338-348, Jan 182011.

[39] L. Wei, et al., "Selective enrichment of $(6,5)$ and $(8,3)$ single-walled carbon nanotubes via cosurfactant extraction from narrow $(n, m)$ distribution samples," Journal of Physical Chemistry B, vol. 112, pp. 2771-2774, Mar 132008.

[40] T. J. McDonald, et al., "Kinetics of PL quenching during single-walled carbon nanotube rebundling and diameter-dependent surfactant interactions," Journal of Physical Chemistry B, vol. 110, pp. 25339-25346, Dec 212006.

[41] S. Ghosh, et al., "Advanced sorting of single-walled carbon nanotubes by nonlinear density-gradient ultracentrifugation," Nature Nanotechnology, vol. 5, pp. 443-450, Jun 2010.

[42] X. M. Tu, et al., "DNA sequence motifs for structure-specific recognition and separation of carbon nanotubes," Nature, vol. 460, pp. 250-253, Jul 92009.

[43] C. Biswas, et al., "Strategy for High Concentration Nanodispersion of SingleWalled Carbon Nanotubes with Diameter Selectivity," Journal of Physical Chemistry C, vol. 113, pp. 10044-10051, Jun 112009.

[44] S. M. Bachilo, et al., "Structure-assigned optical spectra of single-walled carbon nanotubes," Science, vol. 298, pp. 2361-2366, Dec 202002.

[45] E. J. Becraft, et al., "Influence of Alternating L-/D-Amino Acid Chiralities and Disulfide Bond Geometry on the Capacity of Cysteine-Containing Reversible Cyclic Peptides to Disperse Carbon Nanotubes," Biopolymers, vol. 92, pp. 212221, 2009.

[46] M. S. Strano, "Probing chiral selective reactions using a revised Kataura plot for the interpretation of single-walled carbon nanotube spectroscopy," Journal of the American Chemical Society, vol. 125, pp. 16148-16153, Dec 242003.

[47] A. Jorio, et al., "Characterizing carbon nanotube samples with resonance Raman scattering," New Journal of Physics, vol. 5, Oct 162003.

[48] E. K. Hobbie, et al., "Wrinkling and Strain Softening in Single-Wall Carbon Nanotube Membranes," Physical Review Letters, vol. 104, Mar 262010.

[49] T. J. McDonald, et al., "Chiral-selective protection of single-walled carbon nanotube photoluminescence by surfactant selection," Journal of Physical Chemistry C, vol. 111, pp. 17894-17900, Dec 62007.

[50] M. Zheng and E. D. Semke, "Enrichment of single chirality carbon nanotubes," Journal of the American Chemical Society, vol. 129, pp. 6084-+, May 162007.

[51] R. Fleurier, et al., "Transmission Electron Microscopy and UV-vis-IR Spectroscopy Analysis of the Diameter Sorting of Carbon Nanotubes by Gradient Density Ultracentrifugation," Advanced Functional Materials, vol. 19, pp. 22192223, Jul 242009.

[52] R. B. Weisman and S. M. Bachilo, "Dependence of optical transition energies on structure for single-walled carbon nanotubes in aqueous suspension: An empirical Kataura plot," Nano Letters, vol. 3, pp. 1235-1238, Sep 2003. 
[53] V. C. Moore, et al., "Individually suspended single-walled carbon nanotubes in various surfactants," Nano Letters, vol. 3, pp. 1379-1382, Oct 2003.

[54] Z. R. Li, et al., "Spectroscopic Characteristics of Differently Produced SingleWalled Carbon Nanotubes," Chemphyschem, vol. 10, pp. 2296-2304, Sep 92009.

[55] F. Hennrich, et al., "Raman spectroscopy of individual single-walled carbon nanotubes from various sources," Journal of Physical Chemistry B, vol. 109, pp. 10567-10573, Jun 22005.

[56] D. L. Smith, Thin-film deposition : principles and practice. New York: McGrawHill, 1995.

[57] X. Yu, et al., "Fabrication of carbon nanotube based transparent conductive thin films using layer-by-layer technology," Surface \& Coatings Technology, vol. 202, pp. 2002-2007, Feb 152008.

[58] L. Hu, et al., "Percolation in transparent and conducting carbon nanotube networks," Nano Letters, vol. 4, pp. 2513-2517, Dec 2004.

[59] Z. C. Wu, et al., "Transparent, conductive carbon nanotube films," Science, vol. 305, pp. 1273-1276, Aug 272004.

[60] D. Simien, et al., "Influence of nanotube length on the optical and conductivity properties of thin single-wall carbon nanotube networks," Acs Nano, vol. 2, pp. 1879-1884, Sep 2008.

[61] B. B. Parekh, et al., "Improved conductivity of transparent single-wall carbon nanotube thin films via stable postdeposition functionalization," Applied Physics Letters, vol. 90, Mar 192007.

[62] M. Grujicic, et al., "A computational analysis of the percolation threshold and the electrical conductivity of carbon nanotubes filled polymeric materials," Journal of Materials Science, vol. 39, pp. 4441-4449, Jul 152004.

[63] A. V. Kyrylyuk, et al., "Controlling electrical percolation in multicomponent carbon nanotube dispersions," Nature Nanotechnology, vol. 6, pp. 364-369, Jun 2011.

[64] A. Nogales, et al., "Low percolation threshold in nanocomposites based on oxidized single wall carbon nanotubes and poly(butylene terephthalate)," Macromolecules, vol. 37, pp. 7669-7672, Oct 52004. 